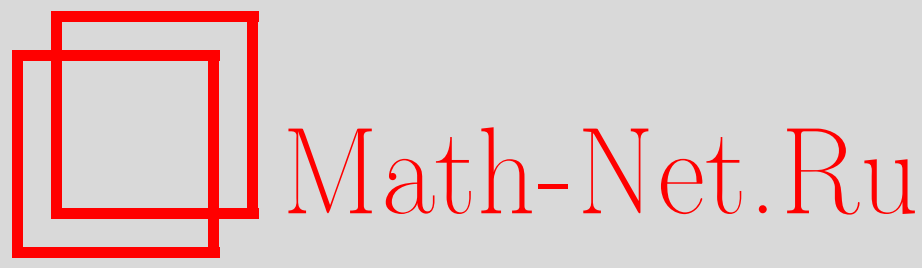

П. И. Дунин-Барковский, А. В. Слепцов, Геометрический гамильтонов формализм для репараметризационно-инвариантных систем с высшими производными, ТМФ, 2009, том 158, номер 1, 72-97

DOI: https://doi.org/10.4213/tmf6300

Использование Общероссийского математического портала Math-Net.Ru подразумевает, что вы прочитали и согласны с пользовательским соглашением http://www.mathnet.ru/rus/agreement

Параметры загрузки:

IP : 3.80 .253 .173

26 апреля 2023 г., 13:02:12

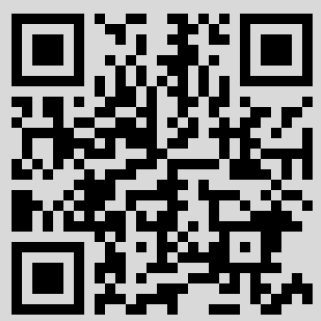




\title{
ГЕОМЕТРИЧЕСКИЙ ГАМИЛЬТОНОВ ФОРМАЛИЗМ ДЛЯ РЕПАРАМЕТРИЗАЦИОННО-ИНВАРИАНТНЫХ СИСТЕМ С ВЫСШИМИ ПРОИЗВОДНЫМИ
}

\begin{abstract}
Рассмотрены репараметризационно-инвариантные лагранжевы системы с высшими производными. Описаны геометрические структуры, появляющиеся в этих теориях, и построен геометрический гамильтонов формализм. Приведена формула преобразования Лежандра для таких систем, которая отличается от обычной. Показано, что фазовое расслоение, т.е. образ преобразования Лежандра, являющееся подмногообразием некоторого кокасательного расслоения, всегда в данной конструкции нечетномерно. Вследствие этого канонически определенная симплектическая 2-форма из объемлющего кокасательного расслоения порождает на фазовом расслоении поле направлений, состоящее из векторов, на которых обращается в нуль ее ограничение. Доказано, что интегральные линии данного поля проецируются на экстремали действия на конфигурационном многообразии. Вышесказанное означает, что полученное поле является гамильтоновым полем. Обнаружено, что соответствующие уравнения Гамильтона записываются через обобщенную скобку Намбу.
\end{abstract}

Ключевые слова: гамильтонов формализм, касательное расслоение высшего порядка, скобка Намбу, репараметризационная инвариантность.

\section{1. ВВЕДЕНИЕ}

Обычная классическая механика рассматривает системы с невырожденными лагранжианами, зависящими только от координат и их первых производных. Условие невырожденности означает, что все скорости могут быть выражены через импульсы, которые получены в результате соответствующего преобразования Лежандра. Это позволяет переформулировать теорию как гамильтонову теорию, что необходимо для канонического квантования. За всем за этим стоят определенные геометрические структуры (см. классический труд [1], а также [2] в качестве учебника по симплектической геометрии и [3] для ее современных приложений). На сегодняшний день геометрический подход к механике является активно развивающейся областью [4].

* Институт теоретический и экспериментальной физики, Москва, Россия. E-mail: barkovs@itep.ru, sleptsov@itep.ru 
Теории с высшими производными изучены меньше, но также важны в физике [5]. Для этих теорий также можно строить гамильтонов формализм и преобразование Лежандра [6]. Подобное построение для вырожденных систем с высшими производными общего вида изложено в [7]. Тем не менее естественного геометрического описания для таких теорий не существует.

Среди всех вырожденных систем выделяется один особый случай - репараметризационно-инвариантные системы. Они представляют интерес в том числе вследствие того, что репараметризационная инвариантность является частным случаем калибровочной инвариантности, которая возникает при описании релятивистских частиц или струн. Интересные мысли о репараметризационно-инвариантных полевых теориях можно найти в [8]. Мы обнаружили,что именно в этом случае репараметризационной инвариантности - существует естественный геометрический способ сконструировать гамильтонов формализм для теорий с высшими производными, который дает формулу преобразования Лежандра, отличающуюся от обычно используемой в общем случае [6], [7].

Обычный геометрический подход к классической механике нельзя просто применить к репараметризационно-инвариантным системам. Основная проблема состоит в следующем. Из-за репараметризационной инвариантности существует непрерывное семейство начальных данных задачи Коши уравнений Эйлера-Лагранжа, каждая точка которого определяет одну и ту же экстремаль на конфигурационном многообразии. Сам факт существования такого семейства представляет собой вырождение, которое необходимо снять. С этой целью мы строим некоторое пространство $\mathcal{P}_{m}$ над каждой точкой $m$ конфигурационного многообразия $\mathcal{M}$. Между всеми точками $\mathcal{P}_{m}$ и всеми экстремалями, проходящими через $m$, должно быть, грубо говоря, взаимно однозначное соответствие. Пространство $\mathcal{P}=\bigcup_{m \in \mathcal{M}} \mathcal{P}_{m}$ мы называем фазовым расслоением. На $\mathcal{P}$ должно существовать поле, интегральные линии которого проецируются в экстремали действия на $\mathcal{M}$. Это поле называется гамильтоновым. Отображение из пространства начальных данных задачи Коши в $\mathcal{P}$ называется преобразованием Лежандра. Привычные формулы для этого преобразования (см. [6], [7]) не удовлетворяют описанному выше требованию: они не снимают вырождение.

В настоящей работе представлено корректно определенное преобразование Лежандра, удовлетворяющее нашему требованию. Также выведены уравнения Гамильтона. Эти результаты получены геометрическим методом. Основную роль в работе играет следующий факт: каждая кривая на конфигурационном многообразии имеет выделенную параметризацию - параметризацию действием.

В конструкции, предложенной в нашей работе, гамильтоново поле возникает естественным образом из геометрических свойств пространства $\mathcal{P}$ как поле направлений, вдоль которых обращается в нуль симплектическая 2-форма (при ограничении на $\mathcal{P}$ ). Уравнения этого поля записаны через обобщенную скобку Намбу (о скобке Намбу см. работы [9], [10]).

Кратко, главными результатами нашей статьи являются нетривиальные формулы для преобразования Лежандра и уравнения Гамильтона, выраженные в терминах обобщенной скобки Намбу. 
Опишем структуру статьи. В разделе 2 обсуждается репараметризационная инвариантность. В разделе 3 мы разбираем простой и хорошо известный случай систем с первыми производными (он изложен также, например, в [11]). В разделе 4 излагается общая конструкция, а в разделе 5 мы изучаем ее подробно на примере систем со вторыми производными. В разделе 6 описана связь симплектической формы с обобщенной скобкой Намбу, используемой в предыдущих разделах. В статье разобраны конкретные примеры.

\section{2. РЕПАРАМЕТРИЗАЦИОННО-ИНВАРИАНТНЫЕ СИСТЕМЫ}

В этом разделе описываются общие свойства репараметризационно-инвариантных систем. Сначала мы рассмотрим случай лагранжианов, зависящих только от координат и их первых производных, а потом уже изучим общий случай.

\section{1. Репараметризационно-инвариантные системы с первыми производ-} ными. Сначала введем понятие репараметризационно-инвариантной лагранжевой системы.

Рассмотрим дифференцируемое многообразие $\mathcal{M}$ и функцию $L$, определенную на его касательном расслоении, т.е. $L: T \mathcal{M} \rightarrow \mathbb{R}$. Назовем $\mathcal{M}$ конфигурационным многообразием, а $L$ - функцией Лагранжа. Обозначим через $\widetilde{T M}$ многообразие, получающееся в результате исключения нулевого сечения из $T \mathcal{M}$. Тогда $L$ является гладкой функцией на многообразии $\widetilde{T \mathcal{M}}$, будучи продолженной на нулевое сечение. Пара $(\mathcal{M}, L)$ называется лагранжевой системой.

Пусть $\gamma:[a, b] \rightarrow \mathcal{M}$ - гладкая кривая на $\mathcal{M}$. Рассмотрим интеграл

$$
S(\gamma)=\int_{a}^{b} L(\gamma(t), \dot{\gamma}(t)) d t
$$

где $t$ - это параметр вдоль кривой, а $\dot{\gamma}(t)$ - касательный вектор к кривой в точке $\gamma(t)$. Данный интеграл называется действием на кривой.

Лагранжева система называется репараметризационно-инвариантной, если действие не зависит от параметризации на кривой, а зависит от кривой только как от множества на $\mathcal{M}$ с фиксированной ориентацией. Другими словами, предположим, что $\gamma_{1}:[a, b] \rightarrow \mathcal{M}, \gamma_{2}:[c, d] \rightarrow \mathcal{M}$ - две произвольно параметризованные кривые в $\mathcal{M}$, так что $\gamma_{1}(a)=\gamma_{2}(c), \gamma_{1}(b)=\gamma_{2}(d), \gamma_{1}([a, b])=\gamma_{2}([a, b])$, т.е. $\gamma_{1}, \gamma_{2}$ - две параметризации одной и той же кривой в $\mathcal{M}$ как ориентированного одномерного подмногообразия. Тогда система называется репараметризационно-инвариантной, если для всех таких кривых выполняется равенство $S\left(\gamma_{1}\right)=S\left(\gamma_{2}\right)$. Чтобы упростить терминологию, будем называть лагранжианы репараметризационно-инвариантными, если они соответствуют репараметризационно-инвариантным системам.

Заметим, что в некотором смысле репараметризационно-инвариантные лагранжианы напоминают дифференциальные 1-формы. Действительно, дифференциальная 1-форма на $\mathcal{M}$ - это функция на $T \mathcal{M}$, которую можно проинтегрировать по кривой на $\mathcal{M}$, причем значение интеграла не будет зависеть от выбранной параметризации на кривой. Разница состоит лишь в том, что дифференциальные 
1-формы - это линейные функции, а репараметризационно-инвариантные лагранжианы линейными функциями не являются. Тем не менее мы увидим ниже, что одним из свойств линейных функций такие лагранжианы все же обладают.

Заметим, что в координатах скорости относительно преобразования переменной $t$ меняются следующим образом:

$$
\dot{x}^{i} \mapsto \frac{1}{f^{\prime}(t)} \dot{x}^{i}, \quad \text { где } \quad t \mapsto f(t) .
$$

Определим, какие ограничения на лагранжианы налагает репараметризационная инвариантность. Пусть $\gamma_{1}:[a, b] \rightarrow \mathcal{M}$ - параметризованная кривая. Зафиксируем гладкую монотонно возрастающую функцию $f:[a, b] \rightarrow \mathbb{R}$. Пусть $[c, d] \subset \mathbb{R}$ - образ функции $f$. Тогда параметризованную кривую $\gamma_{2}:[c, d] \rightarrow \mathcal{M}$ можно получить следующим образом: $\gamma_{2}(\nu)=\gamma_{1}\left(f^{-1}(\nu)\right), \nu \in[c, d]$, т.е. $\gamma_{2}$ является репараметризацией кривой $\gamma_{1}$ с помощью функции $f$. Теперь напишем выражения для действий для обеих кривых:

$$
\begin{aligned}
S\left(\gamma_{1}\right) & =\int_{a}^{b} L\left(\gamma_{1}(\mu), \frac{d}{d \mu} \gamma_{1}(\mu)\right) d \mu \\
S\left(\gamma_{2}\right) & =\int_{c}^{d} L\left(\gamma_{2}(\nu), \frac{d}{d \nu} \gamma_{2}(\nu)\right) d \nu=\int_{f(a)}^{f(b)} L\left(\gamma_{2}(f(\mu)), \frac{1}{f^{\prime}} \frac{d \gamma_{2}}{d \mu}(f(\mu))\right) d f(\mu)= \\
& =\int_{a}^{b} L\left(\gamma_{1}(\mu), \frac{1}{f^{\prime}} \frac{d}{d \mu} \gamma_{1}(\mu)\right) f^{\prime} d \mu .
\end{aligned}
$$

Так как из конструкции следует, что $\gamma_{1}$ и $\gamma_{2}$ совпадают в $\mathcal{M}$ как множества, т.е. $\gamma_{1}([a, b])=\gamma_{2}([c, d])$, условие репараметризационной инвариантности подразумевает, что $S\left(\gamma_{1}\right)=S\left(\gamma_{2}\right)$. Следовательно,

$$
\int_{a}^{b} L\left(\gamma_{1}(\mu), \frac{d}{d \mu} \gamma_{1}(\mu)\right) d \mu=\int_{a}^{b} L\left(\gamma_{1}(\mu), \frac{1}{f^{\prime}} \frac{d}{d \mu} \gamma_{1}(\mu)\right) f^{\prime} d \mu .
$$

Поскольку $f$ - произвольная функция, а $\gamma_{1}$ - произвольная кривая, получаем следующее соотношение для лагранжиана:

$$
L(m, v)=\alpha L\left(m, \frac{v}{\alpha}\right)
$$

для всех $m \in \mathcal{M}, v \in T_{m} \mathcal{M}, \alpha>0$. Переопределяя $\alpha$ как $1 / \alpha$ и переписывая выражение (2.5) в координатах, получим

$$
L\left(x^{i}, \alpha v^{i}\right)=\alpha L\left(x^{i}, v^{i}\right),
$$

где $x^{i}$ - некоторые координаты на $\mathcal{M}$, а $v^{i}$ - координаты на $T_{m} \mathcal{M}$, индуцированные естественным образом координатами $x^{i}$. Это означает, что $L$ - однородная функция первой степени по скоростям. Это свойство может быть записано также с помощью равенства Эйлера:

$$
L=v^{i} \frac{\partial L}{\partial v^{i}} .
$$


Следовательно, несмотря на то что одно свойство линейности для репараметризационно-инвариантных лагранжианов не выполняется, $L\left(v_{1}+v_{2}\right) \neq L\left(v_{1}\right)+L\left(v_{2}\right)$, другое выполняется всегда: $L(\alpha v)=\alpha L(v)$. Значит, для интегрирования 1-формы по кривой действительно важным является только одно свойство линейности. Итак, репараметризационно-инвариантные лагранжианы можно представлять себе как "нелинейную дифференциальную 1-форму" 1).

ПримеР 1. Наиболее простым примером репараметризационно-инвариантного лагранжиана в двумерном евклидовом пространстве является $L=\sqrt{\dot{x}^{2}+\dot{y}^{2}}$. Значение действия на кривой равно просто длине этой кривой.

\section{2. Репараметризационно-инвариантные системы с высшими произ-} водными. Чтобы построить лагранжев формализм с высшими производными, сначала изучим расслоения $k$-го порядка, касательные к дифференцируемому многообразию.

Рассмотрим многообразие $\mathcal{M}$ и зафиксируем число $k \in \mathbb{N}$. Зафиксируем точку $m \in \mathcal{M}$. Рассмотрим все параметризованные кривые на $\mathcal{M}$, которые проходят через $m$. Введем отношение эквивалентности на них. Скажем, что две кривые $\gamma_{1}:[a, b] \rightarrow \mathcal{M}$ и $\gamma_{2}:[c, d] \rightarrow \mathcal{M}, \gamma_{1}\left(\mu_{0}\right)=\gamma_{2}\left(\nu_{0}\right)=m$, эквивалентны, если в некоторой карте на $\mathcal{M}$ все их производные вплоть до $k$-го порядка совпадают в точке $m$. Очевидно, что введенное отношение действительно является отношением эквивалентности и не зависит от выбора карты.

Множество классов эквивалентности относительно введенного выше отношения называется касателъным пространством $k$-го порядка $\kappa \mathcal{M}$ в точке $m$ и обозначается $T_{m}^{k} \mathcal{M}$. Все вместе пространства $T_{m}^{k} \mathcal{M}$, взятые во всех точках многообразия $\mathcal{M}$, образуют расслоение $T^{k} \mathcal{M}$. Таким образом, утверждение, что лагранжиан зависит от производных вплоть до $k$-го порядка, означает, что лагранжиан определен на $T^{k} \mathcal{M}$.

Итак, лагранжевой системой с $k$-ми производными называется пара $(\mathcal{M}, L)$, где $L: T^{k} \mathcal{M} \rightarrow \mathbb{R}$ является гладкой функцией на $\widehat{T^{k} \mathcal{M}}$. Под $\widehat{T^{k} \mathcal{M}}$ мы понимаем многообразие, получающееся в результате исключения нулевого сечения из $T^{k} \mathcal{M}$. Нулевое сечение $T^{k} \mathcal{M}$ определено как класс эквивалентности кривых, у которых все производные обращаются в нуль в соответствующих точках. Заметим, что пространство $T_{m}^{k} \mathcal{M}$ не является векторным пространством для всех $k>1$, в отличие от случая $k=1$.

Действие для систем с высшими производными определяется следующим образом:

$$
S(\gamma)=\int_{a}^{b} L\left(C_{\gamma(t)}(\gamma)\right) d t
$$

где $C_{\gamma(t)}(\gamma) \in T_{\gamma(t)}^{k} \mathcal{M}$ - класс эквивалентности кривой $\gamma$ в точке $\gamma(t)$.

\footnotetext{
1) Однако лагранжианы, обладающие свойством $L(\alpha v)=\alpha L(v)$ не только для $\alpha>0$, но и для $\alpha<0$ (например, $L=\sqrt[3]{x^{3}+y^{3}}$ ), требуют отдельного внимательного рассмотрения, потому что преобразование Лежандра, о котором речь пойдет ниже, не различает ориентации кривых в этом случае. В нашей работе подобные случаи не рассматриваются.
} 
Репараметризационно-инвариантные лагранжевы системы с высшими производными определяются так же, как и в случае первых производных. Система $(\mathcal{M}, L)$ называется репараметризационно-инвариантной, если действие на кривой не зависит от ее параметризации, или, другими словами, для любых двух параметризованных кривых, совпадающих как одномерные ориентированные подмногообразия на $\mathcal{M}$, действие одно и то же.

Таким же образом, каким было получено соотношение (2.6), можно получить аналогичные соотношения для лагранжианов с высшими производными. Например, в случае вторых производных приходим к соотношению

$$
L\left(x^{i}, \alpha v^{i}, \alpha^{2} w^{i}+\beta v^{i}\right)=\alpha L\left(x^{i}, v^{i}, w^{i}\right),
$$

где $\left(x^{i}, v^{i}, w^{i}\right)$ - координаты на $T^{2} \mathcal{M}$, индуцированные естественным образом некоторыми координатами $x^{i}$ на $\mathcal{M}$, а $\alpha>0, \beta$ - произвольные константы. Координаты $v^{i}$ соответствуют первым производным, а $w^{i}$ - вторым. Это условие может быть переписано в виде системы из двух уравнений

$$
L=v^{i} \frac{\partial L}{\partial v^{i}}+2 w^{i} \frac{\partial L}{\partial w^{i}}, \quad v^{i} \frac{\partial L}{\partial w^{i}}=0 .
$$

Данные уравнения называются условиями Цермело [12].

Заметим, что пространство $T_{m}^{2} \mathcal{M}$ не является векторным пространством. Тогда выражение $L\left(x^{i}, \alpha v^{i}, \alpha^{2} w^{i}+\beta v^{i}\right)$ может быть плохо определенным, потому что точка $\left(x^{i}, \alpha v^{i}, \alpha^{2} w^{i}+\beta v^{i}\right)$ может зависеть от выбора координатной карты на $\mathcal{M}$. Действительно, рассмотрим две точки $\xi_{1}, \xi_{2} \in T_{m}^{2} \mathcal{M}$. Введем на $\mathcal{M}$ некоторые координаты $x^{i}$, тогда на $T_{m}^{2} \mathcal{M}$ индуцируются координаты $\left(v^{i}, w^{i}\right)$. Пусть $\xi_{1}, \xi_{2}$ выражаются в этих координатах как $\left(v_{1}^{i}, w_{1}^{i}\right),\left(v_{2}^{i}, w_{2}^{i}\right)$. Тогда, поскольку $T_{m}^{2} \mathcal{M}$ не является линейным пространством, выражение $\left(\alpha v_{1}^{i}+\beta v_{2}^{i}, \alpha w_{1}^{i}+\beta w_{2}^{i}\right)$, вообще говоря, определяет разные точки в $T_{m}^{2} \mathcal{M}$ для одних и тех же $\xi_{1}, \xi_{2}, \alpha, \beta$, если на $\mathcal{M}$ были выбраны разные начальные карты. Однако непосредственной проверкой можно убедиться, что выражение $\left(x^{i}, \alpha v^{i}, \alpha^{2} w^{i}+\beta v^{i}\right)$ задает одну и ту же точку в $T_{m}^{2} \mathcal{M}$ для всех координатных карт. Следовательно, выражение в равенстве (2.9) корректно определено.

ПримеР 2. Приведем самый простой пример репараметризационно-инвариантных лагранжианов со вторыми производными:

$$
L=\frac{(\ddot{x} \dot{y}-\dot{x} \ddot{y})^{2}}{\left(\dot{x}^{2}+\dot{y}^{2}\right)^{5 / 2}} .
$$

Это двумерный евклидов случай релятивистского лагранжиана

$$
L d t=\left(\frac{d^{2} x^{\mu}}{d s^{2}}\right)^{2} d s=\frac{\ddot{x}^{\mu} \ddot{x}_{\mu} \dot{x}^{\nu} \dot{x}_{\nu}-\left(\ddot{x}^{\mu} \dot{x}_{\mu}\right)^{2}}{\left(\dot{x}^{\lambda} \dot{x}_{\lambda}\right)^{5 / 2}} d t,
$$

где $s$ - собственное время. Этот лагранжиан (точнее, слагаемое в лагранжиане) встречается в теории излучения [13]-[15]. Лагранжиан с данным слагаемым, а именно лагранжиан для релятивистской частицы с кривизной, также рассматривается, например, в [16]. 


\section{3. КОНСТРУКЦИЯ ДЛЯ СИСТЕМ \\ С ПЕРВЫМИ ПРОИЗВОДНЫМИ}

Начнем с построения конструкции гамильтонова формализма в простейшем случае, когда лагранжиан зависит от первых производных.

Рассмотрим лагранжеву систему с первыми производными. Координаты на $\mathcal{M}$ обозначим через $x^{i}, i=1, \ldots, n$. Тогда на $T \mathcal{M}$ индуцируются координаты $\left(x^{i}, v^{i}\right)$, а на $T^{*} \mathcal{M}$ - координаты $\left(x^{i}, p_{i}\right)$.

Определим преобразование Лежандра $\mathfrak{L}: T \mathcal{M} \rightarrow T^{*} \mathcal{M}$, где $T \mathcal{M}$ - касательное расслоение $\mathcal{M}$, а $T^{*} \mathcal{M}$ - кокасательное расслоение, следующим образом:

$$
\mathfrak{L}:\left(x^{i}, v^{i}\right) \mapsto\left(x^{i}, \frac{\partial L}{\partial v^{i}}\right) .
$$

Заметим, что $\partial L / \partial v^{i}$ преобразуются при преобразовании координат $x_{i}$ так же, как и $p_{i}$. Следовательно, отображение корректно определено.

Рассмотрим дифференциал преобразования Лежандра. Для фиксированной точки $(m, v) \in T \mathcal{M}$ это линейное отображение $T_{(m, v)}(T \mathcal{M}) \rightarrow T_{\mathfrak{L}(m, v)}\left(T^{*} \mathcal{M}\right)$. Его матрица в базисе, индуцированном координатами, имеет вид

$$
\left(\begin{array}{cc}
\delta_{j}^{i} & 0 \\
\frac{\partial^{2} L}{\partial v^{i} \partial x^{j}} & \frac{\partial^{2} L}{\partial v^{i} \partial v^{j}}
\end{array}\right) .
$$

Нижний левый блок не влияет на ранг матрицы. Дифференцируя уравнение (2.7) по $v^{i}$, получаем связь на нижний правый блок:

$$
v^{i} \frac{\partial^{2} L}{\partial v^{i} \partial v^{j}}=0 .
$$

Так как во всех репараметризационно-инвариантных лагранжианах существует эта связь, то для них $\operatorname{rank} d \mathfrak{L}_{(m, v)} \leqslant 2 n-1$. Ограничимся в дальнейшем рассмотрением только репараметризационно-инвариантных систем общего вида, т.е. без дополнительного вырождения в лагранжиане. Это означает, что

$$
\operatorname{rank} d \mathfrak{L}=2 n-1 .
$$

Заметим, что точки $T \mathcal{M}$ можно понимать как начальные условия задачи Коши уравнений Эйлера-Лагранжа. Это означает, что данная точка из $T \mathcal{M}$ определяет единственное решение уравнения Эйлера-Лагранжа, т.е. вектор в некоторой точке $\mathcal{M}$ задает единственную экстремаль действия, проходящую через данную точку. Очевидно, что в репараметризационно-инвариантном случае все ненулевые векторы, пропорциональные друг другу с положительным коэффициентом пропорциональности, задают одну и ту же непараметризованную ориентированную кривую.

Обозначим образ $\mathfrak{L}$ через $\mathcal{P}$. Он называется фазовым расслоением. Из равенства (2.6), в данном случае записанном как

$$
L(m, \alpha v)=\alpha L(m, v)
$$


следует, что отображение $\mathfrak{L}$ принимает одно и то же значение на каждом векторе $(m, \alpha v), \alpha>0$, где $m \in \mathcal{M}, v \in T \mathcal{M}$. Значит, $\operatorname{dim} \mathcal{P}=2 n-1$. Заметим, что это однопараметрическое семейство $(m, \alpha v)$ в точности является вышеупомянутым множеством точек, которые задают одну и ту же экстремальную кривую.

Так как $\mathcal{P}$ является гиперповерхностью в $T^{*} \mathcal{M}$ (т.е. $(2 n-1)$-мерным подмногообразием), оно может быть задано как решение уравнения $\Phi\left(x^{i}, p_{i}\right)=0$, где $\Phi\left(x^{i}, p_{i}\right)-$ некоторая гладкая функция на $T^{*} \mathcal{M}$. Функция $\Phi\left(x^{i}, p_{i}\right)$ называется связъю. На $T^{*} \mathcal{M}$ как на любом кокасательном расслоении существует каноническая симплектическая 2-форма $\omega$. Ограничение формы $\omega$ на $\mathcal{P}$, как можно видеть из (3.2) (образ дифференциала преобразования Лежандра - касательное пространство к $\mathcal{P}$ ), максимально невырожденно, т.е. имеет следующую каноническую форму:

$$
\left(\begin{array}{ccc}
0 & -E & 0 \\
E & 0 & 0 \\
0 & 0 & 0
\end{array}\right)
$$

Следовательно, как и любая максимально невырожденная дифференциальная 2 -форма на нечетномерном многообразии, она определяет поле направлений на $\mathcal{P}$ (см. п. 4.2). Если $\mathcal{P}$ определено уравнением $\Phi=0$, то можно написать явные формулы для интегральной линии этого поля:

$$
\dot{x}^{i}=c \frac{\partial \Phi}{\partial p_{i}}, \quad \dot{p}_{i}=-c \frac{\partial \Phi}{\partial x^{i}},
$$

где $c$ - произвольный параметр на кривой. Эту систему уравнений будем называть уравнениями Гамильтона. Их можно переписать в следующем виде:

$$
\dot{x}^{i}=c\left\{x^{i}, \Phi\right\}, \quad \dot{p}_{i}=c\left\{p_{i}, \Phi\right\},
$$

где $\{\cdot, \cdot\}$ - обычная скобка Пуассона для функций на кокасательном расслоении.

Уравнения Лагранжа можно вывести из уравнений Гамильтона. Заметим, что

$$
\Phi\left(x^{i}, \frac{\partial L}{\partial v^{i}}\left(x^{i}, v^{i}\right)\right)=0, \quad L=\frac{\partial L}{\partial v^{i}} v^{i} .
$$

Следовательно,

$$
\frac{d}{d t} \frac{\partial L}{\partial v^{i}}=\dot{p}_{i}=-c \frac{\partial \Phi}{\partial x^{i}}=c \frac{\partial^{2} L}{\partial v^{j} \partial x^{i}} \frac{\partial \Phi}{\partial p_{j}}=\frac{\partial^{2} L}{\partial v^{j} \partial x^{i}} \dot{x}^{j}=\frac{\partial L}{\partial v^{j} \partial x^{i}} v^{j}=\frac{\partial L}{\partial x^{i}},
$$

т.е. мы получаем уравнения Лагранжа.

В разделе 4 мы построим преобразование Лежандра и гамильтоново поле в общем случае с высшими производными. Выше была рассмотрена схема построения гамильтонова поля в случае первых производных. Для лучшего понимания рассмотрим также построение преобразования Лежандра в этом простом случае, т.е. получим известную формулу (3.1) тем способом, которым далее будет получено ее обобщение в случае высших производных.

Рассмотрим вектор $v$ в некоторой точке $m_{0} \in \mathcal{M}$. Он определяет экстремальную кривую $\gamma$, проходящую через $m_{0}$. Зафиксируем точку $е$ на кривой $\gamma$ не далеко от $m_{0}$. 
Пусть точка $e$ выбрана на стороне кривой $\gamma$, противоположной тому направлению, в котором указывает $v$ (причины такого выбора см. ниже). В окрестности точки $m_{0}$ определим функцию $\sigma$ следующим образом: значение $\sigma$ в некоторой точке $m$ есть значение действия на единственной экстремальной кривой, которая соединяет $e$ и $m$ (здесь используется тот факт, что для двух данных точек на $\mathcal{M}$ локально существует единственная экстремаль, которая их соединяет). Далее рассмотрим дифференциал функции $\sigma$ в точке $m_{0}:\left.d \sigma\right|_{m_{0}} \in T_{m_{0}}^{*} \mathcal{M}$. Определим преобразование Лежандра следующим образом:

$$
\mathfrak{L}:\left(m_{0}, v\right) \mapsto\left(m_{0},\left.d \sigma\right|_{m_{0}}\right),
$$

где $\left(m_{0}, v\right) \in T \mathcal{M}$ и $\left(m_{0},\left.d \sigma\right|_{m_{0}}\right) \in T^{*} \mathcal{M}$.

Чтобы получить эту формулу в координатах, рассмотрим вариацию действия $S$ на кривой $\gamma$ :

$$
\delta S=S(\gamma+h)-S(\gamma)
$$

где $\gamma+h$ - малое отклонение от кривой $\gamma, h$ - кривая в координатном пространстве $\mathbb{R}^{n}$. Теперь введем некоторую параметризацию на кривых $\gamma$ и $h$ :

$$
\gamma:[a, b] \rightarrow \mathcal{M}, \quad h:[a, b] \rightarrow \mathbb{R}^{n}, \quad \gamma(a)=e, \quad \gamma(b)=m_{0}, \quad h(a)=0 .
$$

Условие $h(a)=0$ соответствует варьированию только одного конца кривой $\gamma$, а именно $m_{0}$. Тогда можно записать

$$
\delta S=\int_{a}^{b}\left(L\left(\gamma^{i}+h^{i}, \dot{\gamma}^{i}+\dot{h}^{i}\right)-L\left(\gamma^{i}, \dot{\gamma}^{i}\right)\right) d t .
$$

Оставляя только члены первого порядка по $h$ и $\dot{h}$, получим

$$
\delta S=\int_{a}^{b}\left(h^{i} \frac{\partial L}{\partial x^{i}}\left(\gamma^{i}(t), \dot{\gamma}^{i}(t)\right)+\dot{h}^{i} \frac{\partial L}{\partial v^{i}}\left(\gamma^{i}(t), \dot{\gamma}^{i}(t)\right)\right) d t
$$

где $\left(x^{i}, v^{i}\right)$ - координаты на $T \mathcal{M}$. Интегрируя второе слагаемое в подынтегральной функции по частям, получим

$$
\begin{aligned}
\delta S= & \left.\left(h^{i}(t) \frac{\partial L}{\partial v^{i}}\left(\gamma^{i}(t), \dot{\gamma}^{i}(t)\right)\right)\right|_{a} ^{b}+ \\
& \quad+\int_{a}^{b}\left(h^{i}(t) \frac{\partial L}{\partial x^{i}}\left(\gamma^{i}(t), \dot{\gamma}^{i}(t)\right)-h^{i}(t) \frac{d}{d t} \frac{\partial L}{\partial v^{i}}\left(\gamma^{i}(t), \dot{\gamma}^{i}(t)\right)\right) d t= \\
= & \left.\left(h^{i}(t) \frac{\partial L}{\partial v^{i}}\left(\gamma^{i}(t), \dot{\gamma}^{i}(t)\right)\right)\right|_{a} ^{b}+ \\
& \quad+\int_{a}^{b}\left(\frac{\partial L}{\partial x^{i}}\left(\gamma^{i}(t), \dot{\gamma}^{i}(t)\right)-\frac{d}{d t} \frac{\partial L}{\partial v^{i}}\left(\gamma^{i}(t), \dot{\gamma}^{i}(t)\right)\right) h^{i}(t) d t
\end{aligned}
$$

Вспомним теперь, что выражение $\frac{\partial L}{\partial x^{i}}\left(\gamma^{i}(t), \dot{\gamma}^{i}(t)\right)-\frac{d}{d t} \frac{\partial L}{\partial v^{i}}\left(\gamma^{i}(t), \dot{\gamma}^{i}(t)\right)$, которое имеет вид левой части уравнения Эйлера-Лагранжа, обращается в нуль для всех $t$, так как 
$\gamma$ - экстремаль действия. Также заметим, что в силу условия $h(a)=0$ только один граничный член вносит вклад. Следовательно, наконец, получаем

$$
\delta S=h^{i}(b) \frac{\partial L}{\partial v^{i}}\left(\gamma^{i}(b), \dot{\gamma}^{i}(b)\right)
$$

Значит, из определения функции $\sigma$ имеем

$$
\left.d \sigma\right|_{m_{0}}=\frac{\partial L}{\partial v^{i}}\left(\gamma^{i}(b), \dot{\gamma}^{i}(b)\right) d x^{i} .
$$

Как было упомянуто, функции $\partial L / \partial v^{i}$ постоянны на всех точках $T_{m_{0}} \mathcal{M}$, определяющих одну и ту же экстремаль. Кривая $\gamma$ определялась как экстремальная кривая, соответствующая вектору $v$, т.е. в произвольной параметризации выполняется равенство

$$
\dot{\gamma}(b)=\alpha v
$$

для некоторого положительного параметра $\alpha$. Параметр $\alpha$ положителен, потому что точка $е$ была выбрана на стороне кривой $\gamma$, противоположной той, куда указывает вектор $v$. Следовательно,

$$
\frac{\partial L}{\partial v^{i}}\left(\gamma^{i}(b), \dot{\gamma}^{i}(b)\right)=\frac{\partial L}{\partial v^{i}}\left(x_{0}^{i}, v_{0}^{i}\right)
$$

где $\left(x_{0}^{i}, v_{0}^{i}\right)$ - координаты точки $\left(m_{0}, v\right) \in T \mathcal{M}$. Итак, получаем

$$
\left.d \sigma\right|_{m_{0}}=\frac{\partial L}{\partial v^{i}}\left(x_{0}^{i}, v_{0}^{i}\right) d x^{i} .
$$

Значит, формула для преобразования Лежандра принимает ожидаемый вид (3.1).

ПримеР 3. Рассмотрим вышеизложенную конструкцию для упомянутого в примере 1 лагранжиана $L=\sqrt{\dot{x}^{2}+\dot{y}^{2}}$ на плоскости $\mathbb{R}^{2}=\mathcal{M}$.

Формула для дифференциала $\sigma$ может быть получена непосредственно, без изучения вариации действия. Пусть для простоты точка $m_{0}$ - начало координат. Пусть вектор $v$ имеет координаты $\left(v_{x 0}, v_{y 0}\right)$. Экстремаль, соответствующая вектору $v$, представляет собой просто прямую линию $\gamma$ в $\mathbb{R}^{2}$, проходящую через начало координат в направлении, указанном вектором $v$. Пусть точка $e$ имеет координаты $\left(x_{0}, y_{0}\right)$. Точка $e$ должна лежать на луче $\gamma$ в противоположной стороне от $v$. Имеем $x_{0} / y_{0}=v_{x 0} / v_{y 0}$, причем коэффициент пропорциональности между $\left(x_{0}, y_{0}\right)$ и $\left(v_{x 0}, v_{y 0}\right)$ имеет отрицательное значение. В силу того что действием на кривой в этом случае является просто длина кривой, а сами экстремали - прямые линии, функцию $\sigma$ можно записать так:

$$
\sigma(x, y)=\sqrt{\left(x-x_{0}\right)^{2}+\left(y-y_{0}\right)^{2}} .
$$

Следовательно, ее дифференциал в точке $m_{0}$ имеет вид

$$
\begin{aligned}
\left.d \sigma\right|_{0} & =-\frac{x_{0}}{\sqrt{x_{0}^{2}+y_{0}^{2}}} d x-\frac{y_{0}}{\sqrt{x_{0}^{2}+y_{0}^{2}}} d y=\frac{v_{x}}{\sqrt{v_{x}^{2}+v_{y}^{2}}} d x+\frac{v_{y}}{\sqrt{v_{x}^{2}+v_{y}^{2}}} d y= \\
& =\frac{\partial L}{\partial \dot{x}}\left(v_{x}, v_{y}\right) d x+\frac{\partial L}{\partial \dot{y}}\left(v_{x}, v_{y}\right) d y .
\end{aligned}
$$


Итак, формула для преобразования Лежандра принимает вид

$$
\mathfrak{L}\left(x, y, v_{x}, v_{y}\right)=\left(x, y, \frac{\partial L}{\partial \dot{x}}\left(x, y, v_{x}, v_{y}\right), \frac{\partial L}{\partial \dot{y}}\left(x, y, v_{x}, v_{y}\right)\right) .
$$

Благодаря тому что преобразование Лежандра определено как

$$
\left(x, y, v_{x}, v_{y}\right) \mapsto\left(x, y, \frac{v_{x}}{\sqrt{v_{x}^{2}+v_{y}^{2}}}, \frac{v_{y}}{\sqrt{v_{x}^{2}+v_{y}^{2}}}\right),
$$

его образ определен уравнением $p_{x}^{2}+p_{y}^{2}=1$, т.е. связь имеет вид $\Phi=p_{x}^{2}+p_{y}^{2}-1$. Уравнения Гамильтона таковы:

$$
\dot{x}=2 c p_{x}, \quad \dot{y}=2 c p_{y}, \quad \dot{p}_{x}=0, \quad \dot{p}_{y}=0 .
$$

Очевидно, что решениями этих уравнений являются всевозможные прямые на плоскости, что и предполагалось.

\section{4. ОБЩАЯ КОНСТРУКЦИЯ}

4.1. Преобразование Лежандра. В этом разделе рассматривается общий случай лагранжевых систем с производными $k$-го порядка. Определим преобразование Лежандра $\mathfrak{L}: T^{2 k-1} \mathcal{M} \rightarrow T^{*} T^{k-1} \mathcal{M}$.

Пусть $m_{2}$ - точка в $T^{2 k-1} \mathcal{M}$. Так как для систем с $k$-м порядком производных уравнения Эйлера-Лагранжа имеют порядок $2 k$, пространство $T_{m}^{2 k-1} \mathcal{M}$ над некоторой точкой $m \in \mathcal{M}$ может рассматриваться как пространство начальных данных задачи Коши для уравнений Эйлера-Лагранжа в этой точке. Следовательно, существует единственная экстремальная кривая $\gamma$ на $\mathcal{M}$, которую можно поднять в $T^{2 k-1} \mathcal{M}$ и которая проходит через точку $m_{2}$ (поднятие кривой в касательное расслоение некоторого порядка означает введение параметризации на этой кривой и отображение кривой в ее производные (производные относительно введенной параметризации)). Заметим, что на каждой кривой существует одна выделенная параметризация - параметризация действием вдоль этой кривой ${ }^{2)}$. Этот факт является центральным в нашей конструкции.

Рассмотрим эту естественную параметризацию действием на кривой $\gamma$. Это позволяет нам поднять $\gamma$ на $T^{k-1} \mathcal{M}$. Заметим, что здесь рассматривается пространство $T^{k-1} \mathcal{M}$, а не $T^{2 k-1} \mathcal{M}$. Пространство $T^{2 k-1} \mathcal{M}$ является пространством начальных данных задачи Коши, в то время как $T^{k-1} \mathcal{M}$ является пространством данных для граничной задачи, т.е. если зафиксировать две достаточно близкие точки на $T^{k-1} \mathcal{M}$, то существует единственная экстремальная кривая на конфигурационном многообразии, поднятие которой соединяет эти две точки. Пусть $m_{1}-$ точка, полученная поднятием $m_{0}$ на $T^{k-1} \mathcal{M}$ с использованием естественной параметризации $\gamma$, где $m_{0} \in \mathcal{M}$ - проекция $m_{2}$ на $\mathcal{M}$. Выберем произвольную точку $e$ на $\widetilde{\gamma}$, близкую к $m_{1}$, где $\widetilde{\gamma}$ - упомянутое поднятие кривой $\gamma$. Пусть точка $e$ выбрана на стороне кривой $\widetilde{\gamma}$, противоположной к той, куда указывает $m_{2}$. Это нужно

\footnotetext{
2) Мы неявно предполагаем здесь, что лагранжиан не обращается в нуль на ненулевых элементах из $T^{k} \mathcal{M}$. В этом случае действие всегда может быть использовано как параметр. В других случаях как параметр действие можно использовать только локально.
} 
для того, чтобы касательный вектор к кривой $\gamma_{e m_{1}}$ в точке $m_{1}$ указывал в том же направлении, что и $m_{2}$.

Определим функцию $\sigma$ в некоторой малой окрестности $U$ точки $m_{1}$ следующим образом. Пусть $r$ - точка из этой окрестности $U$. Тогда существует единственная экстремальная кривая, которая соединяет $e$ и $r$. Определим значение функции $\sigma$ в точке $r$ просто как значение действия на этой кривой, т.е. $\sigma(r)=S\left(\gamma_{e r}\right)$, где $\gamma_{e r}$ - единственная экстремальная кривая, которая может быть поднята в $T^{k-1} \mathcal{M}$, с точками $e$ и $r$ на концах. Определив таким образом функцию $\sigma$ на $U$, можно рассмотреть ее дифференциал $p=\left.d \sigma\right|_{m_{1}}$ в точке $m_{1}$. Заметим, что $p \in T_{m_{1}}^{*} T^{k-1} \mathcal{M}$.

Итак, преобразование Лежандра определено следующим образом:

$$
\left.\mathfrak{L}\left(m_{2}\right) \stackrel{\text { def }}{=} d \sigma\right|_{m_{1}} .
$$

Заметим, что все точки в $T^{2 k-1} \mathcal{M}$, которые определяют одну и ту же экстремальную кривую, как начальные данные задачи Коши при преобразовании Лежандра, отображаются в одну точку. Действительно, единственной информацией о точке из $T^{2 k-1} \mathcal{M}$, которую мы используем в нашей конструкции, является знание о том, какую кривую эта точка определяет в качестве данных Коши.

Теперь укажем способ, которым можно получить явные формулы для преобразования Лежандра. Заметим, что мы до сих пор не показали, что преобразование Лежандра корректно определено. Действительно, оно могло бы зависеть от выбора точки $е$. Однако из явных формул видно, что это не так, и, значит, преобразование корректно определено.

Рассмотрим кривую $\gamma$ как кривую с точками $e_{0}, m_{0}$ на концах, где $e_{0}-$ проекция $e$ на $\mathcal{M}$. На этой кривой $\gamma$ мы будем рассматривать произвольную параметризацию, $\gamma:[a, b] \rightarrow \mathcal{M}, \gamma(a)=e_{0}, \gamma(b)=m_{0}$. Запишем действие на $\gamma:$

$$
S(\gamma)=\int_{a}^{b} L\left(C_{\gamma(t)}^{k}(\gamma)\right) d t
$$

где $C_{\gamma(t)}^{k}(\gamma) \in T_{\gamma(t)}^{k} \mathcal{M}$ - класс эквивалентности кривой $\gamma$ в точке $\gamma(t)$. В координатах действие записывается следующим образом:

$$
S(\gamma)=\int_{a}^{b} L\left(\gamma^{i}, \dot{\gamma}^{i}, \ddot{\gamma}^{i}, \ldots, \stackrel{(k)}{\gamma}^{i}\right) d t
$$

Рассмотрим в координатах на $\mathcal{M}$ малое отклонение от кривой $\gamma-$ кривую $\gamma+h$, где $h:[a, b] \rightarrow \mathbb{R}^{n}, h(a)=0, \dot{h}(a)=0, \ldots, \stackrel{(k-1)}{h}=0$. Пусть $\left(x^{i}, x_{(1)}^{i}, \ldots, x_{(2 k-1)}^{i}\right)-$ координаты на $T^{2 k-1} \mathcal{M}$. Запишем вариацию действия $S$ :

$$
\begin{aligned}
\delta S= & S(\gamma+h)-S(\gamma)=\int_{a}^{b}\left(L\left(\gamma^{i}+h^{i}, \dot{\gamma}^{i}+\dot{h}^{i}, \ddot{\gamma}^{i}+\ddot{h}^{i}, \ldots, \stackrel{(k)^{i}}{\gamma}+\stackrel{(k)}{h} i\right)-\right. \\
& \left.\quad-L\left(\gamma^{i}, \dot{\gamma}^{i}, \ddot{\gamma}^{i}, \ldots, \stackrel{(k)}{\gamma} i\right)\right) d t= \\
= & h^{i}(b)\left(\frac{\partial L}{\partial x_{(1)}^{i}}-\frac{d}{d t} \frac{\partial L}{\partial x_{(2)}^{i}}+\cdots+(-1)^{k-1} \frac{d^{k-1}}{d t^{k-1}} \frac{\partial L}{\partial x_{(k)}^{i}}\right)+
\end{aligned}
$$




$$
\begin{array}{r}
+\dot{h}^{i}(b)\left(\frac{\partial L}{\partial x_{(2)}^{i}}-\frac{d}{d t} \frac{\partial L}{\partial x_{(3)}^{i}}+\cdots+(-1)^{k-2} \frac{d^{k-2}}{d t^{k-2}} \frac{\partial L}{\partial x_{(k)}^{i}}\right)+\cdots \\
\cdots+h^{i}(b) \frac{\partial L}{\partial x_{(k)}^{i}},
\end{array}
$$

где оператор производной $d / d t$, примененный к функциям на $T^{2 k-1} \mathcal{M}$, имеет вид

$$
\frac{d}{d t}=x_{(1)}^{i} \frac{\partial}{\partial x^{i}}+x_{(2)}^{i} \frac{\partial}{\partial x_{(1)}^{i}}+\cdots+x_{(2 k-1)}^{i} \frac{\partial}{\partial x_{(2 k-2)}^{i}} .
$$

Все выражения в правой части (4.4) взяты в точке $C_{\gamma(b)}^{2 k-1}(\gamma) \in T_{\gamma(b)}^{2 k-1} \mathcal{M}$, представляющий собой класс эквивалентности произвольно параметризованной кривой $\gamma$ в точке $m_{0}$ (более подробно о формуле (4.4) см. приложение А).

Теперь вернемся к параметризации действием кривой $\gamma$ и из определения функции $\sigma$ получим выражение для ее дифференциала в точке $m_{1}$ :

$$
\begin{aligned}
&\left.d \sigma\right|_{m_{1}}=\left(\frac{\partial L}{\partial x_{(1)}^{i}}-\frac{d}{d t} \frac{\partial L}{\partial x_{(2)}^{i}}+\cdots+(-1)^{k-1} \frac{d^{k-1}}{d t^{k-1}} \frac{\partial L}{\partial x_{(k)}^{i}}\right) d x+ \\
&+\left(\frac{\partial L}{\partial x_{(2)}^{i}}-\frac{d}{d t} \frac{\partial L}{\partial x_{(3)}^{i}}+\cdots+(-1)^{k-2} \frac{d^{k-2}}{d t^{k-2}} \frac{\partial L}{\partial x_{(k)}^{i}}\right) d x_{(1)}^{i}+\cdots \\
& \cdots+\frac{\partial L}{\partial x_{(k)}^{i}} d x_{(k-1)}^{i}
\end{aligned}
$$

где все выражения взяты в точке $C_{m_{0}}^{2 k-1}(\gamma) \in T_{m_{0}}^{k} \mathcal{M}$, представляющей собой класс эквивалентности кривой $\gamma$, параметризованной действием, в точке $m_{0}$. Мы видим, что выражение для $d \sigma$ не зависит от выбора точки $e$, и, следовательно, преобразование Лежандра корректно определено.

Чтобы переписать формулу для преобразования Лежандра через координаты $\left(y^{i}, y_{(1)}^{i}, \ldots, y_{(2 k-1)}^{i}\right)$ начальной точки $m_{2} \in T^{2 k-1} \mathcal{M}$, сначала необходимо найти координаты точки $C_{m_{0}}^{2 k-1}(\gamma)$. Поскольку рассматривалась параметризация действием кривой $\gamma$, выражение для них имеет следующий вид:

$$
\left(\gamma^{i}\left(b_{0}\right), \frac{d \gamma^{i}}{d s}\left(b_{0}\right), \ldots, \frac{d^{k} \gamma^{i}}{d s^{k}}\left(b_{0}\right)\right)
$$

где $b_{0}=S\left(\gamma_{e m_{1}}\right)$, т.е. $\gamma\left(b_{0}\right)=m_{1}$. Заметим, что $\frac{d \gamma}{d s}=\frac{1}{L} \frac{d \gamma}{d t}$, где лагранжиан $L$ взят в соответствующей точке. Обозначим лагранжиан $L$, взятый в этой точке, через $L_{0}$, $\frac{d L}{d t}-$ через $\dot{L}_{0}$ и т.д. Итак, координаты $C_{m_{0}}^{2 k-1}(\gamma)$ переписываются следующим образом:

$$
\left(y^{i}, \frac{1}{L_{0}} y_{(1)}^{i}, \frac{1}{L_{0}^{2}} y_{(2)}^{i}-\frac{\dot{L}_{0}}{L_{0}^{3}} y_{(1)}^{i}, \frac{1}{L_{0}^{3}} y_{(3)}^{i}-3 \frac{\dot{L}_{0}}{L_{0}^{4}} y_{(2)}^{i}+\left(3 \frac{\dot{L}_{0}^{2}}{L_{0}^{5}}-\frac{\ddot{L}_{0}}{L_{0}^{4}}\right) y_{(1)}^{i}, \ldots\right)
$$


Все эти координаты получаются раскрытием по правилу Лейбница выражений вида

$$
\frac{1}{L} \frac{d}{d t} \frac{1}{L} \frac{d}{d t} \cdots \frac{1}{L} \frac{d}{d t} \gamma^{i}
$$

для каждой из них, а затем взятием результата в соответствующей точке.

Окончательно формула для преобразования Лежандра получается подстановкой координат точки $C_{m_{0}}^{2 k-1}(\gamma)$ в выражение для $\left.d \sigma\right|_{m_{1}}$. Используя соотношения вида (2.6), (2.9) (высшие условия Цермело) для функции Лагранжа с $k$-ми производными, можно вынести множители $L_{0}, \dot{L}_{0}, \ldots$ из аргументов функций. В случае, например, вторых производных в итоге получается следующая формула для преобразования Лежандра:

$$
\left(x^{i}, x_{(1)}^{i}, x_{(2)}^{i}, x_{(3)}^{i}\right) \mapsto\left(x^{i}, \frac{1}{L} x_{(1)}^{i}, \frac{\partial L}{\partial x_{(1)}^{i}}-\frac{d}{d t} \frac{\partial L}{\partial x_{(2)}^{i}}, L \frac{\partial L}{\partial x_{(2)}^{i}}\right) .
$$

Чтобы сделать все эти шаги более понятными, мы подробно описываем конструкцию на примере случая вторых производных в разделе 5.

Данная конструкция может восприниматься как простое обобщение понятий релятивистской механики. Преобразование Лежандра отображает точку из $T^{2 k-1} \mathcal{M}$ в ковектор из кокасательного пространства к $T^{k-1} \mathcal{M}$. Координаты этой точки в $T^{k-1} \mathcal{M}$ аналогичны 4-скоростям релятивистской механики, так как они получаются как производные кривой в параметризации действием, т.е. "собственным временем". Ковектор $\left.d \sigma\right|_{m_{1}}$ - это просто обобщение и более формальная запись определения импульса $\partial S / \partial x$, используемого в релятивистской механике (см. [17]).

4.2. Гамильтоново поле. Каждое $(2 k-1)$-параметрическое семейство точек из $T^{2 k-1} \mathcal{M}$, которые задают одну и ту же экстремальную кривую, отображается целиком в одну точку, поэтому размерность образа преобразования Лежандра на $2 k-1$ меньше, чем размерность $T^{2 k-1} \mathcal{M}$. Другими словами,

$$
\operatorname{dim}\left(\mathfrak{L}\left(T^{2 k-1} \mathcal{M}\right)\right)=2 k n-(2 k-1)
$$

(мы предполагаем, что лагранжиан вырожден не более, чем это требуется условием репараметризационной инвариантности; более формально: $\operatorname{rank} d \mathfrak{L}=2 k n-(2 k-1)$, см. случай первых производных в разделе 3$)$. Таким образом, многообразие $\mathcal{P}=$ $\mathfrak{L}\left(T^{2 k-1} \mathcal{M}\right) \subset T^{*} T^{k-1} \mathcal{M}$ - нечетномерное подмногообразие. Отметим, что $\mathcal{P}$ можно рассматривать и как расслоение над $T^{k-1} \mathcal{M}$, и как расслоение над $\mathcal{M}$.

На расслоении $T^{*} T^{k-1} \mathcal{M}$, как на любом кокасательном расслоении, задана каноническая симплектическая структура. Ограничение $\omega_{\mathcal{P}}$ симплектической 2-формы $\omega$ на подмногообразие $\mathcal{P}$ как максимально невырожденная (это может быть проверено аналогично случаю первых производных, описанному в разделе 3) дифференциальная 2-форма на нечетномерном многообразии задает в каждой точке $\mathcal{P}$ направление, т.е. некоторое одномерное подпространство касательного пространства (см. раздел 6). Это подпространство $V_{\chi}$ в точке $\chi \in \mathcal{P}$ определяется как подпространство всех векторов $\eta \in T_{\chi} \mathcal{P}$, которые удовлетворяют условию

$$
\omega_{\mathcal{P}}(\eta)=0 .
$$


Так как $\omega_{\mathcal{P}}$ является максимально невырожденной 2-формой на нечетномерном многообразии, $V_{\chi}$ в точности одномерно.

Набор пространств $V_{\chi}$ на всем $\mathcal{P}$ называется гамильтоновым полем. Это поле направлений, а не векторное поле, что согласуется с репараметризационной инвариантностью. Так как ожидается, что проекции интегральных линий этого поля на $\mathcal{M}$ будут экстремалями действия, их параметризация не важна, а поле, интегральные линии которого не параметризованы, - это как раз поле направлений.

Для $V_{\chi}$ можно написать явную формулу используя так называемую обобщенную скобку Намбу ${ }^{3)}$ (см. раздел 6). Пусть $\eta \in V_{\chi}$ - ненулевой вектор, $\eta=\eta^{\mu} \frac{\partial}{\partial \xi^{\mu}}$, где $\xi=(x, p), x^{i}, p^{i}-$ координаты на $T^{*} T^{k-1} \mathcal{M}$. Тогда выполняется следующее равенство:

$$
\eta^{\mu}=\left\{\xi^{\mu}, \Phi_{1}, \ldots, \Phi_{2 k-1}\right\},
$$

где $\{\cdot, \ldots, \cdot\}$ - обобщенная скобка Намбу между $2 k$ функциями (вывод $(4.12)$ и подробнее о скобках Намбу см. ниже в разделе 6).

Теперь докажем, что задача нахождения экстремалей действия сводится к задаче нахождения интегральных кривых гамильтонова поля, как это и ожидалось.

Сначала покажем, что для любой кривой $\gamma$ в $\mathcal{M}$

$$
S(\gamma)=\int_{\gamma_{\mathcal{P}}} \theta_{\mathcal{P}}
$$

Здесь $\gamma_{\mathcal{P}}-$ кривая в $\mathcal{P}$. Она получается поднятием $\gamma$ в $T^{2 k-1} \mathcal{M}$ в некоторой параметризации, а затем применением преобразования Лежандра. Отметим, что при применении преобразования Лежандра образ не зависит от выбора параметризации. Заметим теперь, что проекция $\tilde{\gamma}$ кривой $\gamma_{\mathcal{P}}$ на $T^{k-1} \mathcal{M}(\mathcal{P}-$ это, в частности, расслоение над $\left.T^{k-1} \mathcal{M}\right)$ является в точности поднятием кривой $\gamma$ в то же пространство при использовании параметризации действием. Пусть $\theta_{\mathcal{P}}$ - ограничение канонической 1-формы $\theta$ из $T^{*} T^{k-1} \mathcal{M}$ на $\mathcal{P}$.

Заметим, что по определению канонической 1 -формы в точке $\left(m_{1}, p\right) \in \mathcal{P}$, где $m_{1} \in T^{k-1} \mathcal{M}, p \in T_{m_{1}}^{*} T^{k-1} \mathcal{M}$, выполняется следующее равенство:

$$
\theta_{\mathcal{P}\left(m_{1}, p\right)}(\eta)=p\left(\pi_{\mathcal{P}}^{\prime}(\eta)\right)
$$

где $\eta \in T_{\left(m_{1}, p\right)} \mathcal{P}$, а $\pi_{\mathcal{P}}^{\prime} \in \operatorname{Hom}\left(T_{\left(m_{1}, p\right)} \mathcal{P}, T_{m_{1}} T^{k-1} \mathcal{M}\right)$ - производная отображения $\pi_{\mathcal{P}}$ проекции $\mathcal{P}$ на $T^{k-1} \mathcal{M}$.

Введем некоторую параметризацию на $\gamma_{\mathcal{P}}$ и соответствующую параметризацию на $\tilde{\gamma}$. Таким образом, имеем $\gamma_{\mathcal{P}}:[a, b] \rightarrow \mathcal{P}$ и $\tilde{\gamma}:[a, b] \rightarrow T^{k-1} \mathcal{M}$. По определению интеграла 1-формы по кривой

$$
\int_{\gamma_{\mathcal{P}}} \theta_{\mathcal{P}}=\int_{a}^{b} \theta_{\mathcal{P}}\left(\dot{\gamma}_{\mathcal{P}}(t)\right) d t
$$

где $\dot{\gamma}_{\mathcal{P}}(t)$ - касательный вектор к $\gamma_{\mathcal{P}}$ в точке $\gamma_{\mathcal{P}}(t)$. Заметим, что $\pi_{\mathcal{P}}^{\prime}\left(\dot{\gamma}_{\mathcal{P}}(t)\right)=\dot{\tilde{\gamma}}(t)$, так как $\tilde{\gamma}-$ проекция $\gamma_{\mathcal{P}}$.

3) Авторы благодарны В. Рубцову за предложенную идею рассмотреть скобки Намбу. 
По определению преобразования Лежандра точка $\gamma_{\mathcal{P}}(t)$ - это пара $\left(\tilde{\gamma}(t),\left.d \sigma\right|_{\tilde{\gamma}(t)}\right)$, где $\left.d \sigma\right|_{\tilde{\gamma}(t)} \in T_{\tilde{\gamma}(t)}^{*} T^{k-1} \mathcal{M}$ - дифференциал функции $\sigma$, описанной в п. 4.1. Заметим, что этот дифференциал не зависит от выбора начальной точки $e$, следовательно, без потери общности можно взять в качестве точки $e$ точку $\tilde{\gamma}(a)$. Тогда все точки кривой $\gamma_{\mathcal{P}}$ оказываются получены как дифференциалы одной и той же функции $\sigma$. По определению канонической 1-формы, таким образом, имеем

$$
\theta_{\mathcal{P}}\left(\dot{\gamma}_{\mathcal{P}}(t)\right)=\left.d \sigma\right|_{\tilde{\gamma}(t)}(\dot{\tilde{\gamma}}(t)) .
$$

Но интеграл дифференциала некоторой функции вдоль кривой - это просто разность значений функции в конечных точках:

$$
\left.\int_{a}^{b} d \sigma\right|_{\tilde{\gamma}(t)}(\dot{\tilde{\gamma}}(t)) d t=\int_{\tilde{\gamma}} d \sigma=\sigma(\tilde{\gamma}(b))-\sigma(\tilde{\gamma}(a)) .
$$

По определению $\sigma$ имеем $\sigma(\tilde{\gamma}(a))=0, \sigma(\tilde{\gamma}(b))=S(\tilde{\gamma})$. Таким образом, мы получаем желаемое равенство

$$
S(\gamma)=\int_{\gamma_{\mathcal{P}}} \theta_{\mathcal{P}}
$$

Значит, экстремали действия на многообразии $\mathcal{M}$ являются проекциями экстремалей интеграла ограничения канонической 1-формы на многообразие $\mathcal{P}$. Рассмотрим небольшое отклонение от кривой $\gamma_{\mathcal{P}}-$ кривую $\gamma_{\mathcal{P}^{\prime}}$. Изменение интеграла канонической 1-формы

$$
\int_{\gamma_{\mathcal{P}}} \theta_{\mathcal{P}}-\int_{\gamma_{\mathcal{P}}} \theta_{\mathcal{P}}
$$

равно симплектической площади поверхности, соединяющей кривые $\gamma_{\mathcal{P}}, \gamma_{\mathcal{P}^{\prime}}$ и, следовательно, является бесконечно малым более высокого порядка, чем расстояние между $\gamma_{\mathcal{P}}$ и $\gamma_{\mathcal{P}^{\prime}}$ в том случае, когда $\gamma_{\mathcal{P}}$ - интегральная кривая поля нулевых направлений $\omega_{\mathcal{P}}$ (следствие формулы Стокса; подробнее см., например, [2]).

Таким образом, интегральные линии поля нулевых направлений $\omega_{\mathcal{P}}$ являются экстремалями интеграла $\theta$, а значит, проецируются в экстремали действия на $\mathcal{M}$.

\section{5. КОНСТРУКЦИЯ ДЛЯ СИСТЕМ С ЛАГРАНЖИАНОМ, ЗАВИСЯЩИМ ОТ ВТОРЫХ ПРОИЗВОДНЫХ}

Рассмотрим репараметризационно-инвариантное действие со вторыми производными:

$$
S=\int_{a}^{b} L\left(\gamma^{i}, \dot{\gamma}^{i}, \ddot{\gamma}^{i}\right) d t
$$

Уравнения Эйлера-Лагранжа имеют следующий вид:

$$
\frac{\partial L}{\partial x^{i}}-\frac{d}{d t}\left(\frac{\partial L}{\partial v^{i}}-\frac{d}{d t} \frac{\partial L}{\partial w^{i}}\right)=0,
$$

где $\left(x^{i}, v^{i}, w^{i}\right)$ - координаты на $T^{2} \mathcal{M}, \mathcal{M}$ - конфигурационное многообразие. Это дифференциальные уравнения четвертого порядка, решения которых являются экстремалями функционала (5.1). Выберем точку $m \in \mathcal{M}$. Чтобы определить единственным образом экстремаль, проходящую через $m$, необходимо задать значения 
первой, второй и третьей производных кривой в этой точке. Другими словами, пространством начальных данных задачи Коши является $T_{m}^{3} \mathcal{M}$ (определение $T_{m}^{k} \mathcal{M}$ см. в п. 2.2).

Пусть $m_{2}$ - точка в $T_{m}^{3} \mathcal{M}$, тогда ее выбор однозначно задает экстремальную кривую, проходящую через $m$. Однако любая точка $\left(x_{0}, v_{0}, w_{0}, u_{0}\right) \in T^{3} \mathcal{M}$ принадлежит трехпараметрическому семейству

$$
\left(x_{0}^{i}, \alpha v_{0}^{i}, \alpha^{2} w_{0}^{i}+\beta v_{0}^{i}, \alpha^{3} u_{0}^{i}+3 \alpha \beta w_{0}^{i}+\delta v_{0}^{i}\right),
$$

где каждая точка задает одну и ту же экстремаль; здесь $\alpha>0, \beta, \delta$ - произвольные параметры (см. приложение Б). Требуется определить некоторое пространство $\mathcal{P}_{m}$ над $m$ такое, чтобы существовало взаимно однозначное соответствие между точками этого пространства и множеством всех экстремалей действия, проходящих через $m$. Другими словами, необходимо осуществить такой переход от $T_{m}^{3} \mathcal{M}$ к $\mathcal{P}_{m}$, чтобы устранялось вырождение, проявляющееся в вышеописанных трехпараметрических семействах. Для этого строится отображение

$$
\mathfrak{L}: T^{3} \mathcal{M} \rightarrow T^{*} T \mathcal{M}
$$

где $T^{3} \mathcal{M}=\bigcup_{m \in \mathcal{M}} T_{m}^{3} \mathcal{M}, \mathcal{P}=\bigcup_{m \in \mathcal{M}} \mathcal{P}_{m}, \mathcal{P}=\mathfrak{L}\left(T^{3} \mathcal{M}\right) ; \mathfrak{L}$ называется преобразованием Лежандра, $\mathcal{P}$ - фазовым расслоением.

Пусть точка $m_{2} \in T^{3} \mathcal{M}, m_{0}$ - ее проекция на $\mathcal{M}$, а $\gamma$ - экстремаль на $\mathcal{M}$, соответствующая $m_{2}$ (как решение уравнений Эйлера-Лагранжа с начальными данными, заданными $m_{2}$ ). Заметим, что на каждой кривой имеется выделенная параметризация - параметризация действием. Пусть $\tilde{\gamma}$ - поднятие $\gamma$ в TM с помощью этой параметризации. При этом точка $m_{0} \in \gamma$ поднимается в некоторую точку $m_{1} \in T \mathcal{M}$. Заметим, что пространство $T \mathcal{M}$ можно понимать как пространство данных краевой задачи для уравнений Эйлера-Лагранжа.

Определим теперь в некоторой небольшой окрестности $U$ точки $m_{1}$ функцию $\sigma$ следующим образом. Пусть $r$ - точка в $U$. Тогда в общем положении существует единственное решение краевой задачи для уравнений Эйлера-Лагранжа, которое соединяет точки $e$ и $r$. Определим значение функции $\sigma$ в точке $r$ как значение интеграла действия на этой кривой (проекции на $\mathcal{M})$. Таким образом, $\sigma(r)=S\left(\gamma_{e r}\right)$, где $\gamma_{e r}$ - единственная экстремаль, соединяющая $e$ и $r$. Определив таким образом функцию $\sigma$ на $U$, можно рассмотреть ее дифференциал $p=\left.d \sigma\right|_{m_{1}}$ в точке $m_{1}$. Заметим, что $p \in T_{m_{1}}^{*} T \mathcal{M}$.

Итак, определим преобразование Лежандра как

$$
\mathfrak{L}\left(m_{2}\right):=\left.d \sigma\right|_{m_{1}} .
$$

Все точки семейства (5.3) отображаются в одну и ту же точку. В самом деле, все точки этого семейства задают одну и ту же экстремальную кривую и, следовательно, одну и ту же функцию $\sigma$ и ее дифференциал $d \sigma$.

Выведем явную формулу для преобразования Лежандра. Рассмотрим произвольную параметризацию кривой $\gamma$. Ограничимся рассмотрением отрезка этой кривой 
между точками $e_{0}$ и $m_{0}$, где $e_{0}-$ проекция $e$ на $\mathcal{M}$. Пусть $\gamma+h-$ близкая к $\gamma$ кривая, причем $\gamma:[a, b] \rightarrow \mathcal{M}, \gamma(a)=e_{0}, \gamma(b)=m_{0}, h:[a, b] \rightarrow \mathbb{R}^{n}, h(a)=0, \dot{h}(a)=0$. Рассмотрим вариацию действия $S$

$$
\begin{aligned}
\delta S=S(\gamma+h)-S(\gamma)=( & \left.\frac{\partial L}{\partial v^{i}}\left(\gamma^{i}, \dot{\gamma}^{i}, \ddot{\gamma}^{i}\right)-\left(\frac{d}{d t} \frac{\partial L}{\partial w^{i}}\right)\left(\gamma^{i}, \dot{\gamma}^{i}, \ddot{\gamma}^{i}\right)\right) h^{i}(b)+ \\
& +\frac{\partial L}{\partial w^{i}}\left(\gamma^{i}, \dot{\gamma}^{i}, \ddot{\gamma}^{i}\right) \dot{h}^{i}(b)
\end{aligned}
$$

производная $d / d t$ здесь означает

$$
\frac{d}{d t}=v^{i} \frac{\partial}{\partial x^{i}}+w^{i} \frac{\partial}{\partial v^{i}}+u^{i} \frac{\partial}{\partial w^{i}} .
$$

По определению функции $\sigma$ имеем

$$
\left.d \sigma\right|_{m_{1}}=\left(\frac{\partial L}{\partial v^{i}}\left(C_{m_{0}}^{2}(\gamma)\right)-\left(\frac{d}{d t} \frac{\partial L}{\partial w^{i}}\right)\left(C_{m_{0}}^{3}(\gamma)\right)\right) d x^{i}+\frac{\partial L}{\partial w^{i}}\left(C_{m_{0}}^{2}(\gamma)\right) d v^{i}
$$

где $C_{m_{0}}^{2}(\gamma) \in T_{m_{0}}^{2} \mathcal{M}, C_{m_{0}}^{3}(\gamma) \in T_{m_{0}}^{3} \mathcal{M}$ - соответствующие классы эквивалентности в точке $m_{0}$ кривой $\gamma$, параметризованной действием. Производная $\frac{d}{d t} \frac{\partial L}{\partial w^{i}}$ зависит не только от $x^{i}, v^{i}, w^{i}$, но и от $u^{i}$, т.е. от точки в $T_{m_{0}}^{3} \mathcal{M}$, из-за присутствия коэффициентов $u^{i}$ в формуле (5.7). Мы видим, что выражение для $d \sigma$ не содержит зависимости от $e$, и, следовательно, преобразование Лежандра корректно определено.

Координаты точки $C_{m_{0}}^{3}(\gamma)=C_{\gamma\left(b_{0}\right)}^{3}(\gamma)$, где $b_{0}=S(\gamma)$ (т.е. $\left.\gamma\left(b_{0}\right)=m_{0}\right)$, имеют вид

$$
\left(\gamma^{i}\left(b_{0}\right), \frac{d \gamma^{i}}{d s}\left(b_{0}\right), \frac{d^{2} \gamma^{i}}{d s^{2}}\left(b_{0}\right), \frac{d^{3} \gamma^{i}}{d s^{3}}\left(b_{0}\right)\right)
$$

где $s$ - значение действия вдоль $\gamma$, а координаты $C_{m_{0}}^{2}(\gamma)$ равны соответственно

$$
\left(\gamma^{i}\left(b_{0}\right), \frac{d \gamma^{i}}{d s}\left(b_{0}\right), \frac{d^{2} \gamma^{i}}{d s^{2}}\left(b_{0}\right)\right) .
$$

Следовательно, выражение для $d \sigma$ принимает следующий вид:

$$
\begin{aligned}
\left.d \sigma\right|_{m_{1}}=( & \frac{\partial L}{\partial v^{i}}\left(\gamma^{i}\left(b_{0}\right), \frac{d \gamma^{i}}{d s}\left(b_{0}\right), \frac{d^{2} \gamma^{i}}{d s^{2}}\left(b_{0}\right)\right)- \\
& \left.-\left(\frac{d}{d t} \frac{\partial L}{\partial w^{i}}\right)\left(\gamma^{i}\left(b_{0}\right), \frac{d \gamma^{i}}{d s}\left(b_{0}\right), \frac{d^{2} \gamma^{i}}{d s^{2}}\left(b_{0}\right), \frac{d^{3} \gamma^{i}}{d s^{3}}\left(b_{0}\right)\right)\right) d x^{i}+ \\
& +\frac{\partial L}{\partial w^{i}}\left(\gamma^{i}\left(b_{0}\right), \frac{d \gamma^{i}}{d s}\left(b_{0}\right), \frac{d^{2} \gamma^{i}}{d s^{2}}\left(b_{0}\right)\right) d v^{i}
\end{aligned}
$$

Теперь отметим, что $\frac{d \gamma}{d s}=\frac{1}{L} \frac{d \gamma}{d t}=\frac{\dot{\gamma}}{L}$, где значение функции $L$ взято в соответствующей точке. Значение $L$ в этой точке обозначим через $L_{0}, \frac{d L}{d t}-$ через $\dot{L}_{0}, \frac{d^{2} L}{d t^{2}}-$ через $\ddot{L}_{0}$. Таким образом, координаты точки $C_{m_{0}}^{3}(\gamma)$ переписываются как

$$
\left(\gamma^{i}(b), \frac{\dot{\gamma}^{i}(b)}{L_{0}}, \frac{\ddot{\gamma}^{i}(b)}{L_{0}^{2}}-\frac{\dot{L}_{0}}{L_{0}^{3}} \dot{\gamma}^{i}(b), \frac{1}{L_{0}^{3}} \dddot{\gamma}^{i}(b)-3 \frac{\dot{L}_{0}}{L_{0}^{4}} \ddot{\gamma}^{i}(b)+\left(3 \frac{\dot{L}_{0}^{2}}{L_{0}^{5}}-\frac{\ddot{L}_{0}}{L_{0}^{4}}\right) \dot{\gamma}^{i}(b)\right) .
$$


Используя обозначения $\gamma^{i}(b)=x_{0}^{i}, \dot{\gamma}^{i}(b)=v_{0}^{i}, \ddot{\gamma}^{i}(b)=w_{0}^{i}, \dddot{\gamma}^{i}(b)=u_{0}^{i}$, перепишем (5.12) как

$$
\left(x_{0}^{i}, \frac{v_{0}^{i}}{L_{0}}, \frac{w_{0}^{i}}{L_{0}^{2}}-\frac{\dot{L}_{0}}{L_{0}^{3}} v_{0}^{i}, \frac{1}{L_{0}^{3}} u_{0}^{i}-3 \frac{\dot{L}_{0}}{L_{0}^{4}} w_{0}^{i}+\left(3 \frac{\dot{L}_{0}^{2}}{L_{0}^{5}}-\frac{\ddot{L}_{0}}{L_{0}^{4}}\right) v_{0}^{i}\right) .
$$

Наконец, формула для преобразования Лежандра получается подстановкой этих координат точки $C_{2}(\gamma(b))$ в выражение для $\left.d \sigma\right|_{m_{1}}$ :

$$
\begin{aligned}
\left.d \sigma\right|_{m_{1}}=( & \frac{\partial L}{\partial v^{i}}\left(x_{0}^{i}, \frac{v_{0}^{i}}{L_{0}}, \frac{w_{0}^{i}}{L_{0}^{2}}-\frac{\dot{L}_{0}}{L_{0}^{3}} v_{0}^{i}\right)- \\
& -\left(\frac{d}{d t} \frac{\partial L}{\partial w^{i}}\right)\left(x_{0}^{i}, \frac{v_{0}^{i}}{L_{0}}, \frac{w_{0}^{i}}{L_{0}^{2}}-\frac{\dot{L}_{0}}{L_{0}^{3}} v_{0}^{i}, \frac{1}{L_{0}^{3}} u_{0}^{i}-3 \frac{\dot{L}_{0}}{L_{0}^{4}} w_{0}^{i}+\right. \\
& \left.\left.+\left(3 \frac{\dot{L}_{0}^{2}}{L_{0}^{5}}-\frac{\ddot{L}_{0}}{L_{0}^{4}}\right) v_{0}^{i}\right)\right) d x^{i}+\frac{\partial L}{\partial w^{i}}\left(x_{0}^{i}, \frac{v_{0}^{i}}{L_{0}}, \frac{w_{0}^{i}}{L_{0}^{2}}-\frac{\dot{L}_{0}}{L_{0}^{3}} v_{0}^{i}\right) d v^{i}
\end{aligned}
$$

Рассмотрим соотношение (2.9). Дифференцируя это соотношение по $w^{i}$, получим

$$
\alpha^{2} \frac{\partial L}{\partial w^{i}}\left(x^{i}, \alpha v^{i}, \alpha^{2} w^{i}+\beta v^{i}\right)=\alpha \frac{\partial L}{\partial w^{i}}\left(x^{i}, v^{i}, w^{i}\right)
$$

т.е.

$$
\frac{\partial L}{\partial w^{i}}\left(x^{i}, \alpha v^{i}, \alpha^{2} w^{i}+\beta v^{i}\right)=\frac{1}{\alpha} \frac{\partial L}{\partial w^{i}}\left(x^{i}, v^{i}, w^{i}\right) .
$$

Аналогично получим

$$
\begin{gathered}
\frac{\partial L}{\partial v^{i}}\left(x^{i}, \alpha v^{i}, \alpha^{2} w^{i}+\beta v^{i}\right)-\left(\frac{d}{d t} \frac{\partial L}{\partial w^{i}}\right)\left(x^{i}, \alpha v^{i}, \alpha^{2} w^{i}+\beta v^{i}, \alpha^{3} u^{i}+3 \alpha \beta w^{i}+\epsilon v^{i}\right)= \\
=\frac{\partial L}{\partial v^{i}}\left(x^{i}, v^{i}, w^{i}\right)-\left(\frac{d}{d t} \frac{\partial L}{\partial w^{i}}\right)\left(x^{i}, v^{i}, w^{i}, u^{i}\right) .
\end{gathered}
$$

Используя обозначения $\alpha=1 / L_{0}, \beta=-\dot{L}_{0} / L_{0}^{3}$, получим

$$
\begin{gathered}
\frac{\partial L}{\partial w^{i}}\left(x_{0}^{i}, \alpha v_{0}^{i}, \alpha^{2} w_{0}^{i}+\beta v_{0}^{i}\right)=\frac{1}{\alpha} \frac{\partial L}{\partial w^{i}}\left(x_{0}^{i}, v_{0}^{i}, w_{0}^{i}\right) \\
\left(\frac{\partial L}{\partial v^{i}}-\frac{d}{d t} \frac{\partial L}{\partial w^{i}}\right)\left(x_{0}^{i}, \alpha v_{0}^{i}, \alpha^{2} w_{0}^{i}+\beta v_{0}^{i}, \alpha^{3} u_{0}^{i}+3 \alpha \beta w_{0}^{i}+\epsilon v_{0}^{i}\right)= \\
=\left(\frac{\partial L}{\partial v^{i}}-\frac{d}{d t} \frac{\partial L}{\partial w^{i}}\right)\left(x_{0}^{i}, v_{0}^{i}, w_{0}^{i}, u_{0}^{i}\right)
\end{gathered}
$$

для произвольного $\epsilon$. Следовательно, выражение для $\left.d \sigma\right|_{m_{1}}$ может быть записано каK

$$
\left.d \sigma\right|_{m_{1}}=\left(\frac{\partial L}{\partial v^{i}}\left(x_{0}^{i}, v_{0}^{i}, w_{0}^{i}\right)-\left(\frac{d}{d t} \frac{\partial L}{\partial w^{i}}\right)\left(x_{0}^{i}, v_{0}^{i}, w_{0}^{i}, u_{0}^{i}\right)\right) d x^{i}+L_{0} \frac{\partial L}{\partial w^{i}}\left(x_{0}^{i}, v_{0}^{i}, w_{0}^{i}\right) d v^{i} .
$$


Таким образом, формула для преобразования Лежандра в координатах принимает следующий вид:

$$
\mathfrak{L}:\left(x^{i}, v^{i}, w^{i}, u^{i}\right) \mapsto\left(x^{i}, \frac{v^{i}}{L}, \frac{\partial L}{\partial v^{i}}-\frac{d}{d t} \frac{\partial L}{\partial w^{i}}, L \frac{\partial L}{\partial w^{i}}\right),
$$

где все выражения в правой части взяты в точке $\left(x^{i}, v^{i}, w^{i}, u^{i}\right)$.

Поскольку размерность $\mathcal{P}=\mathfrak{L}\left(T^{3} \mathcal{M}\right)$ равна $4 n-3$, можно задать $\mathcal{P}$ тремя уравнениями

$$
\Phi_{1}\left(x^{i}, a^{i}, p_{i}, s_{i}\right)=0, \quad \Phi_{2}\left(x^{i}, a^{i}, p_{i}, s_{i}\right)=0, \quad \Phi_{3}\left(x^{i}, a^{i}, p_{i}, s_{i}\right)=0,
$$

где $\left(x^{i}, a^{i}, p_{i}, s_{i}\right)$ - координаты на $T^{*} T \mathcal{M}, \Phi_{1}, \Phi_{2}, \Phi_{3}$ - некоторые функции. На $T^{*} T^{1} \mathcal{M}$ имеется каноническая симплектическая 2-форма

$$
\omega=d p_{i} \wedge d x^{i}+d s_{i} \wedge d a^{i}
$$

Для вектора

$$
\dot{x}^{i} \frac{\partial}{\partial x^{i}}+\dot{a}^{i} \frac{\partial}{\partial a^{i}}+\dot{p}^{i} \frac{\partial}{\partial p^{i}}+\dot{s}^{i} \frac{\partial}{\partial s^{i}}=\dot{\xi}^{\mu} \frac{\partial}{\partial \xi^{\mu}}
$$

из нулевого направления $\omega$ на $\mathcal{P}$ имеем

$$
\dot{\xi}^{\mu}=c\left\{\xi^{\mu}, \Phi_{1}, \Phi_{2}, \Phi_{3}\right\},
$$

где $\{\cdot, \cdot, \cdot, \cdot\}$ - обобщенная скобка Намбу от четырех функций, а $c$ - константа (см. раздел 6).

Следовательно, имеем уравнения Гамильтона следующего вида:

$$
\begin{aligned}
& \dot{x}^{i}=c\left(\left\{x^{i}, \Phi_{1}\right\}\left\{\Phi_{2}, \Phi_{3}\right\}+\left\{x^{i}, \Phi_{2}\right\}\left\{\Phi_{3}, \Phi_{1}\right\}+\left\{x^{i}, \Phi_{3}\right\}\left\{\Phi_{1}, \Phi_{2}\right\}\right), \\
& \dot{a}^{i}=c\left(\left\{a^{i}, \Phi_{1}\right\}\left\{\Phi_{2}, \Phi_{3}\right\}+\left\{a^{i}, \Phi_{2}\right\}\left\{\Phi_{3}, \Phi_{1}\right\}+\left\{a^{i}, \Phi_{3}\right\}\left\{\Phi_{1}, \Phi_{2}\right\}\right), \\
& \dot{p}_{i}=c\left(\left\{p_{i}, \Phi_{1}\right\}\left\{\Phi_{2}, \Phi_{3}\right\}+\left\{p_{i}, \Phi_{2}\right\}\left\{\Phi_{3}, \Phi_{1}\right\}+\left\{p_{i}, \Phi_{3}\right\}\left\{\Phi_{1}, \Phi_{2}\right\}\right), \\
& \dot{s}_{i}=c\left(\left\{s_{i}, \Phi_{1}\right\}\left\{\Phi_{2}, \Phi_{3}\right\}+\left\{s_{i}, \Phi_{2}\right\}\left\{\Phi_{3}, \Phi_{1}\right\}+\left\{s_{i}, \Phi_{3}\right\}\left\{\Phi_{1}, \Phi_{2}\right\}\right),
\end{aligned}
$$

где $\{\cdot, \cdot\}$ - скобка Пуассона.

ПримеР 4. Рассмотрим лагранжиан (2.10). Преобразование Лежандра задается KaK

$$
\mathfrak{L}:\left(x, y, v_{x}, v_{y}, w_{x}, w_{y}, u_{x}, u_{y}\right) \mapsto\left(x, y, a_{x}, a_{y}, p_{x}, p_{y}, s_{x}, s_{y}\right),
$$

где

$$
\begin{gathered}
a_{x}=\frac{v_{x}\left(v_{x}^{2}+v_{y}^{2}\right)^{5 / 2}}{\left(w_{x} v_{y}-v_{x} w_{y}\right)^{2}}, \quad a_{y}=\frac{v_{y}\left(v_{x}^{2}+v_{y}^{2}\right)^{5 / 2}}{\left(w_{x} v_{y}-v_{x} w_{y}\right)^{2}}, \\
p_{x}=\left[-4 w_{x} v_{y} v_{x}^{2} w_{y}+6 w_{x} v_{y}^{3} w_{y}+5 w_{x}^{2} v_{y}^{2} v_{x}-v_{x}^{3} w_{y}^{2}-6 v_{x} w_{y}^{2} v_{y}^{2}-\right. \\
\left.-2 v_{y}^{2} u_{x} v_{x}^{2}-2 v_{y}^{4} u_{x}+2 v_{y} v_{x}^{3} u_{y}+2 v_{y}^{3} v_{x} u_{y}\right] /\left(v_{x}^{2}+v_{y}^{2}\right)^{7 / 2}, \\
p_{y}=\left[-\left(6 w_{x}^{2} v_{y} v_{x}^{2}+w_{x}^{2} v_{y}^{3}+4 w_{x} v_{y}^{2} v_{x} w_{y}-6 v_{x}^{3} w_{y} w_{x}-5 v_{x}^{2} w_{y}^{2} v_{y}-\right.\right. \\
\left.\left.-2 v_{x}^{3} u_{x} v_{y}-2 v_{x} u_{x} v_{y}^{3}+2 v_{x}^{4} u_{y}+2 v_{x}^{2} u_{y} v_{y}^{2}\right)\right] /\left(v_{x}^{2}+v_{y}^{2}\right)^{7 / 2},
\end{gathered}
$$




$$
s_{x}=-2 \frac{\left(v_{x} w_{y}-v_{y} w_{x}\right)^{3} v_{y}}{\left(v_{x}^{2}+v_{y}^{2}\right)^{5}}, \quad s_{y}=2 \frac{\left(v_{x} w_{y}-v_{y} w_{x}\right)^{3} v_{x}}{\left(v_{x}^{2}+v_{y}^{2}\right)^{5}} .
$$

Следовательно, многообразие $\mathcal{P}$ в пространстве $T^{*} T \mathcal{M}$ может быть задано системой из трех уравнений

$$
s_{x} v_{x}+s_{y} v_{y}=0, \quad p_{x} v_{x}+p_{y} v_{y}-1=0, \quad\left(v_{x}^{2}+v_{y}^{2}\right)^{5}-16 \frac{v_{x}^{2} v_{y}^{2}}{s_{x}^{2} s_{y}^{2}}=0 .
$$

Введем обозначения

$$
\Phi_{1}=s_{x} v_{x}+s_{y} v_{y}, \quad \Phi_{2}=p_{x} v_{x}+p_{y} v_{y}-1, \quad \Phi_{3}=\left(v_{x}^{2}+v_{y}^{2}\right)^{5}-16 \frac{v_{x}^{2} v_{y}^{2}}{s_{x}^{2} s_{y}^{2}} .
$$

Тогда гамильтоново поле задается следующей скобкой Намбу:

$$
\dot{\xi}^{\mu}=c\left\{\xi^{\mu}, \Phi_{1}, \Phi_{2}, \Phi_{3}\right\}
$$

где $\dot{\xi}^{\mu}-$ компоненты этого поля. Следовательно, уравнения Гамильтона принимают следующий вид:

$$
\begin{aligned}
\dot{x}= & a_{x}\left(\left(10\left(a_{x}^{2}+a_{y}^{2}\right)^{4} a_{x}-32 \frac{a_{x} a_{y}^{2}}{s_{x}^{2} s_{y}^{2}}\right) a_{x}+\left(10\left(a_{x}^{2}+a_{y}^{2}\right)^{4} a_{y}-32 \frac{a_{x}^{2} a_{y}}{s_{x}^{2} s_{y}^{2}}\right) a_{y}-64 \frac{a_{x}^{2} a_{y}^{2}}{s_{x}^{2} s_{y}^{2}}\right), \\
\dot{y}= & a_{y}\left(\left(10\left(a_{x}^{2}+a_{y}^{2}\right)^{4} a_{x}-32 \frac{a_{x} a_{y}^{2}}{s_{x}^{2} s_{y}^{2}}\right) a_{x}+\left(10\left(a_{x}^{2}+a_{y}^{2}\right)^{4} a_{y}-32 \frac{a_{x}^{2} a_{y}}{s_{x}^{2} s_{y}^{2}}\right) a_{y}-64 \frac{a_{x}^{2} a_{y}^{2}}{s_{x}^{2} s_{y}^{2}}\right), \\
\dot{a}_{x}= & a_{x}\left(32 \frac{p_{x} a_{x}^{2} a_{y}^{2}}{s_{x}^{3} s_{y}^{2}}+32 \frac{p_{y} a_{x}^{2} a_{y}^{2}}{s_{x}^{2} s_{y}^{3}}\right)+32 \frac{a_{x}^{2} a_{y}^{2}\left(-p_{x} a_{x}-p_{y} a_{y}\right)}{s_{x}^{3} s_{y}^{2}}, \\
\dot{a}_{y}= & a_{y}\left(32 \frac{p_{x} a_{x}^{2} a_{y}^{2}}{s_{x}^{3} s_{y}^{2}}+32 \frac{p_{y} a_{x}^{2} a_{y}^{2}}{s_{x}^{2} s_{y}^{3}}\right)+32 \frac{a_{x}^{2} a_{y}^{2}\left(-p_{x} a_{x}-p_{y} a_{y}\right)}{s_{x}^{2} s_{y}^{3}}, \\
\dot{p}_{x}= & 0, \\
\dot{s}_{x}= & -s_{x}\left(32 \frac{p_{x} a_{x}^{2} a_{y}^{2}}{s_{x}^{3} s_{y}^{2}}+32 \frac{p_{y} a_{x}^{2} a_{y}^{2}}{s_{x}^{2} s_{y}^{3}}\right)-p_{x}\left(\left(10\left(a_{x}^{2}+a_{y}^{2}\right)^{4} a_{x}-32 \frac{a_{x} a_{y}^{2}}{s_{x}^{2} s_{y}^{2}}\right) a_{x}+\right. \\
& \left.+\left(10\left(a_{x}^{2}+a_{y}^{2}\right)^{4} a_{y}-32 \frac{a_{x}^{2} a_{y}}{s_{x}^{2} s_{y}^{2}}\right) a_{y}-64 \frac{a_{x}^{2} a_{y}^{2}}{s_{x}^{2} s_{y}^{2}}\right)+ \\
& +\left(-10\left(a_{x}^{2}+a_{y}^{2}\right)^{4} a_{x}+32 \frac{a_{x} a_{y}^{2}}{s_{x}^{2} s_{y}^{2}}\right)\left(-p_{x} a_{x}-p_{y} a_{y}\right), \\
& +\left(-10\left(a_{x}^{2}+a_{y}^{2}\right)^{4} a_{y}+32 \frac{a_{x}^{2} a_{y}}{s_{x}^{2} s_{y}^{2}}\right)\left(-p_{x} a_{x}-p_{y} a_{y}\right) . \\
& \left.+\left(10\left(a_{x}^{2}+a_{y}^{2}\right)^{4} a_{y}-32 \frac{a_{x}^{2} a_{y}}{s_{x}^{2} s_{y}^{2}}\right) b-64 \frac{a_{x}^{2} a_{y}^{2}}{s_{x}^{2} s_{y}^{2}}\right)+ \\
\dot{s}_{y}= & -s_{y}\left(32 \frac{p_{x} a_{x}^{2} a_{y}^{2}}{s_{x}^{3} s_{y}^{2}}+32 \frac{p_{y} a_{x}^{2} a_{y}^{2}}{s_{x}^{2} s_{y}^{3}}\right)-p_{y}\left(\left(10\left(a_{x}^{2}+a_{y}^{2}\right)^{4} a_{x}-32 \frac{a_{x} a_{y}^{2}}{s_{x}^{2} s_{y}^{2}}\right) a_{x}+\right. \\
& (-1,
\end{aligned}
$$




\section{6. НУЛЬ-ВЕКТОРЫ СИМПЛЕКТИЧЕСКОЙ ФОРМЫ И ОБОБЩЕННАЯ СКОБКА НАМБУ}

В данном разделе показано, что нуль-векторы ограничения симплектической формы на нечетномерное многообразие определяются обобщенной скобкой Намбу (определение обобщенной скобки Намбу см. например, в [9], [10]).

Рассмотрим $2 n$-мерное симплектическое многообразие $\mathcal{N}$ с симплектической 2 -формой $\omega$. Пусть $\mathcal{P} \subset \mathcal{N}-(2 n-(2 k-1))$-мерное подмногообразие для $k \leqslant n^{4)}$. Пусть также многообразие $\mathcal{P}$ таково, что ограничение $\omega_{\mathcal{P}}$ формы $\omega$ на $\mathcal{P}$ - максимально невырожденная 2-форма. Напомним, что 2-форма на нечетномерном многообразии называется максимально невырожденной, если ее ядро везде одномерно, т.е. она в каждой точке приводится к каноническому виду (3.6), где $E$ - единичная $((n-k) \times(n-k))$-матрица, если $\operatorname{dim} \mathcal{P}=2 n-(2 k-1)$. В каждой точке $m$ многообразия $\mathcal{P}$ существует выделенное одномерное подпространство пространства $T_{m} \mathcal{P}$, на котором $\omega_{\mathcal{P}}$ обращается в нуль.

Поскольку $\mathcal{P}-(2 n-(2 k-1))$-мерное подмногообразие многообразия $\mathcal{N}$, его можно задать системой уравнений

$$
\Phi_{1}=0, \quad \Phi_{2}=0, \quad \ldots, \quad \Phi_{2 k-1}=0
$$

с некоторыми $2 k-1$ функциями $\Phi_{1}, \ldots, \Phi_{2 k-1}$.

Заметим, что симплектическая 2-форма $\omega$ задает отождествление между $T \mathcal{N}$ и $T^{*} \mathcal{N}: \xi \mapsto \omega(\xi)$ для $\xi \in T \mathcal{N}$. Это отождествление также продолжается естественным образом и на все тензорные степени $T \mathcal{N}$ и $T^{*} \mathcal{N}$ соответственно.

Рассмотрим $k$-ю внешнюю степень формы $\omega-2 k$-форму $\omega^{\wedge k} \in \Omega^{2 k} \mathcal{N}=\Lambda^{2 k} T^{*} \mathcal{N}$. Ее образ $\pi_{k}$ в $\Lambda^{2 k} T \mathcal{N}$ при вышеописанном отождествлении определяется следующим образом:

$$
\pi_{k}\left(c_{1}, \ldots, c_{2 k}\right) \stackrel{\text { def }}{=} \omega^{\wedge k}\left(\pi\left(c_{1}\right), \ldots, \pi\left(c_{2 k}\right)\right),
$$

где $c_{i}$ - произвольные ковекторы, а $\pi \in \Lambda^{2} T \mathcal{N}$ - бивектор, соответствующий $\omega$.

Теперь покажем, что вектор $\pi_{k}\left(d \Phi_{1}, \ldots, d \Phi_{2 k-1}\right) \in T_{m} \mathcal{P}$, взятый в некоторой точке $m \in \mathcal{P}$, лежит в вышеописанном выделенном одномерном подпространстве. Это выражение действительно является вектором, поскольку при подстановке $2 k-1$ ковекторов в элемент $\Lambda^{2 k} T \mathcal{N}$ получится вектор, т.е. элемент $T \mathcal{N}$. Так как форма $\omega$ невырожденна и, следовательно, вектор $\pi_{k}$ также невырожден, а формы $d \Phi_{i}, i=1,2, \ldots, 2 k-1$, линейно независимы, полученный вектор ненулевой. Как проверить, что $\pi_{k}\left(d \Phi_{1}, \ldots, d \Phi_{2 k-1}\right)$ - действительно искомый вектор? Во-первых, он должен быть касательным к $\mathcal{P}$, т.е. формы $d \Phi_{i}$ для всех $i$ должны на нем обращаться в нуль. Это действительно так, поскольку

$$
d \Phi_{i}\left(\pi_{k}\left(d \Phi_{1}, \ldots, d \Phi_{2 k-1}\right)\right)=\pi_{k}\left(d \Phi_{1}, \ldots, d \Phi_{2 k-1}, d \Phi_{i}\right)=0
$$

из-за кососимметричности $\pi_{k}$. Во-вторых, ограничение $\omega_{\mathcal{P}}$ формы $\omega$ на $\mathcal{P}$ должно обращаться в нуль на $\mathcal{P}$, т.е. 1-форма, получающаяся в результате применения $\omega$

\footnotetext{
4) Результаты этого раздела используются в настоящей статье для $\mathcal{N}=T^{*} T^{k-1} \mathcal{M}$ и $\mathcal{P}=$ $\mathfrak{L}\left(T^{2 k-1} \mathcal{M}\right)$.
} 
к этому вектору, должна быть линейной комбинацией форм $d \Phi_{i}$. Пусть $\xi-$ некоторый вектор. Тогда

$$
\begin{aligned}
\omega\left(\pi_{k}\left(d \Phi_{1}, \ldots, d \Phi_{2 k-1}\right), \xi\right) & =\pi_{k}\left(d \Phi_{1}, \ldots, d \Phi_{2 k-1}, \omega(\xi)\right)= \\
& =\omega^{\wedge k}\left(\pi\left(d \Phi_{1}\right), \ldots, \pi\left(d \Phi_{2 k-1}\right), \pi(\omega(\xi))\right)= \\
& =\omega^{\wedge k}\left(\pi\left(d \Phi_{1}\right), \ldots, \pi\left(d \Phi_{2 k-1}\right), \xi\right) .
\end{aligned}
$$

Последнее выражение является суммой членов вида $\omega\left(\pi\left(d \Phi_{i}\right), \xi\right)$ с некоторыми коэффициентами, не зависящими от $\xi$. Заметим, что $\omega\left(\pi\left(d \Phi_{i}\right), \xi\right)=\omega\left(\pi\left(d \Phi_{i}\right)\right)(\xi)=$ $d \Phi_{i}(\xi)$. Значит, $\omega\left(\pi_{k}\left(d \Phi_{1}, \ldots, d \Phi_{2 k-1}\right)\right)$ - линейная комбинация форм $d \Phi_{i}$.

Тензор $\pi_{k}$ задает скобку на наборах $2 k$ функций на $\mathcal{N}$ следующим образом:

$$
\left\{f_{1}, \ldots, f_{2 k}\right\} \stackrel{\text { def }}{=} \pi_{k}\left(d f_{1}, \ldots, d f_{2 k}\right) .
$$

По определению $\pi_{k}$ имеем

$$
\left\{f_{1}, \ldots, f_{2 k}\right\}=\sum_{\sigma} \frac{\operatorname{sign} \sigma}{2^{n} n !}\left\{f_{\sigma_{1}}, f_{\sigma_{2}}\right\} \ldots\left\{f_{\sigma_{2 k-1}}, f_{\sigma_{2 k}}\right\},
$$

где суммирование ведется по всем перестановкам $\sigma$ для $1, \ldots, 2 k ;\{\cdot, \cdot\}$ - обычная скобка Пуассона, порождаемая $\omega$. Это в точности совпадает с определением обобщенной скобки Намбу (см. формулу (8) п. 2.2 в [10]).

Следовательно, если вектор из выделенного подпространства имеет вид $\dot{\zeta_{\mu}} \frac{\partial}{\partial \zeta_{\mu}}$, где $\zeta_{\mu}-$ координаты на $\mathcal{N}$, то его компоненты записываются следующим образом:

$$
\dot{\zeta_{\mu}}=c\left\{\zeta_{\mu}, \Phi_{1}, \ldots, \Phi_{2 k-1}\right\},
$$
где $c$ - константа, одинаковая во всех выражениях для $\dot{\zeta_{\mu}}$, a $\{\underbrace{\cdot, \ldots,}_{2 k}\}-$ обобщенная
скобка Намбу от $2 k$ функций.

\section{7. ЗАКЛЮЧЕНИЕ}

В работе построен гамильтонов формализм для репараметризационно-инвариантных систем с производными порядка $k$. Основная идея состоит в том, чтобы рассматривать выделенную параметризацию на кривой - параметризацию действием. Используя этот факт, можно построить корректно определенное преобразование Лежандра $\mathfrak{L}: T^{2 k-1} \mathcal{M} \rightarrow T^{*} T^{k-1} \mathcal{M}$. Оказывается, что его образ при этом всегда является нечетномерным многообразием, на котором в силу нечетномерности возникает естественным образом поле направлений (гамильтоново поле). Для любого конкретного $k$ можно записать уравнения Гамильтона, эквивалентные уравнениям Лагранжа.

Отметим, что этот результат имеет физическую интерпретацию. Преобразование Лежандра отвечает переходу от описания теории в переменных "координаты-скорости" к описанию в переменных "координаты-импульсы". Приведенные в данной работе формулы для координат и импульсов похожи на соответствующие формулы релятивистской механики. Это происходит вследствие того, что релятивистская механика является репараметризационно-инвариантной теорией. 
Многие вопросы, однако, все еще требуют ответа. Как применить результаты механики Намбу в рамках данного формализма? Как построить квантовую теорию в соответствующих терминах? Как формализм обобщается, если рассматривать вложения в конфигурационное многообразие не линий, а поверхностей? Как он связан с теорией поля и теорией струн? Было бы интересно исследовать эти вопросы.

\section{ПРИЛОЖКНИЕ А}

\section{Вариация действия}

Здесь выводится стандартная формула для вариации действия, используемая в П. 4.1 ,

$$
\begin{aligned}
\delta S=S(\gamma+h)-S(\gamma)=\int_{a}^{b} & \left(L\left(\gamma^{i}+h^{i}, \dot{\gamma}^{i}+\dot{h}^{i}, \ddot{\gamma}^{i}+\ddot{h}^{i}, \ldots, \stackrel{(k)}{\gamma} i+\stackrel{(k)}{h} i\right)-\right. \\
& \left.-L\left(\gamma^{i}, \dot{\gamma}^{i}, \ddot{\gamma}^{i}, \ldots \stackrel{(k)}{\gamma} \text { i }\right)\right) d t
\end{aligned}
$$

Оставляя только члены первого порядка по $h$ и $\dot{h}$, имеем

$$
\delta S=\int_{a}^{b}\left(h^{i} \frac{\partial L}{\partial x^{i}}\left(\gamma^{i}, \ldots, \stackrel{(k)}{\gamma} i\right)+\dot{h}^{i} \frac{\partial L}{\partial x_{(1)}^{i}}\left(\gamma^{i}, \ldots, \stackrel{(k)}{\gamma} i\right)+\cdots+\stackrel{(k)}{h} i \frac{\partial L}{\partial x_{(k)}^{i}}\left(\gamma^{i}, \ldots, \stackrel{(k)}{\gamma} i\right)\right) d t
$$

Интегрируя по частям все члены под интегралом, кроме первого, получаем

$$
\begin{aligned}
& \delta S=\left(h^{i}(t) \frac{\partial L}{\partial x_{(1)}^{i}}\left(\gamma^{i}(t), \ldots, \stackrel{(k)}{\gamma} i(t)\right)+\dot{h}^{i}(t) \frac{\partial L}{\partial x_{(2)}^{i}}\left(\gamma^{i}(t), \ldots, \stackrel{(k)}{\gamma} i(t)+\ldots\right.\right. \\
&\left.\ldots+\stackrel{(k-1)}{h}{ }^{i}(t) \frac{\partial L}{\partial x_{(k)}^{i}}\left(\gamma^{i}(t), \ldots, \stackrel{(k)}{\gamma} i(t)\right)\right)\left.\right|_{a} ^{b}+\int_{a}^{b}\left(h^{i} \frac{\partial L}{\partial x^{i}}\left(\gamma^{i}, \ldots, \stackrel{(k)}{\gamma} i\right)-\right. \\
&\left.-h^{i} \frac{d}{d t}\left(\frac{\partial L}{\partial x_{(1)}^{i}}\left(\gamma^{i}, \ldots, \stackrel{(k)}{\gamma} i\right)+\cdots+\stackrel{(k)}{h} \frac{\partial L}{\partial x_{(k)}^{i}}\left(\gamma^{i}, \ldots, \stackrel{(k)}{\gamma} i\right)\right)\right) d t .
\end{aligned}
$$

Заметим, что поскольку $h(a)=0, \dot{h}(a)=0, \ldots, \stackrel{(k-1)}{h}=0$, половина граничных членов обращается в нуль. Таким образом, выражение (А.3) переписывается как

$$
\begin{array}{r}
\delta S=h^{i}(b) \frac{\partial L}{\partial x_{(1)}^{i}}\left(\gamma^{i}(b), \ldots, \stackrel{(k)}{\gamma} i(b)\right)+\dot{h}^{i}(b) \frac{\partial L}{\partial x_{(2)}^{i}}\left(\gamma^{i}(b), \ldots, \stackrel{(k)}{\gamma} i(b)\right)+\cdots \\
\ldots+\stackrel{(k-1)}{h^{i}}(b) \frac{\partial L}{\partial x_{(k)}^{i}}\left(\gamma^{i}(b), \ldots, \stackrel{(k)}{\gamma} i(b)\right)+\int_{a}^{b}\left(h^{i} \frac{\partial L}{\partial x^{i}}\left(\gamma^{i}, \ldots, \stackrel{(k)}{\gamma} i\right)-\right. \\
\left.-h^{i} \frac{d}{d t}\left(\frac{\partial L}{\partial x_{(1)}^{i}}\left(\gamma^{i}, \ldots, \stackrel{(k)}{\gamma} i\right)+\cdots+\stackrel{(k)}{h} \frac{\partial L}{\partial x_{(k)}^{i}}\left(\gamma^{i}, \ldots, \stackrel{(k)}{\gamma} i\right)\right)\right) d t .
\end{array}
$$


Повторяя аналогичное интегрирование по частям начиная с третьего члена под интегралом, в итоге получаем

$$
\begin{aligned}
\delta S=h^{i}(b) & \left(\frac{\partial L}{\partial x_{(1)}^{i}}-\frac{d}{d t} \frac{\partial L}{\partial x_{(2)}^{i}}+\cdots+(-1)^{k-1} \frac{d^{k-1}}{d t^{k-1}} \frac{\partial L}{\partial x_{(k)}^{i}}\right)+ \\
& +\dot{h}^{i}(b)\left(\frac{\partial L}{\partial x_{(2)}^{i}}-\frac{d}{d t} \frac{\partial L}{\partial x_{3}^{i}}+\cdots+(-1)^{k-2} \frac{d^{k-2}}{d t^{k-2}} \frac{\partial L}{\partial x_{(k)}^{i}}\right)+\cdots+h^{i}(b) \frac{\partial L}{\partial x_{(k)}^{i}}+ \\
& +\int_{a}^{b} h^{i}\left(\frac{\partial L}{\partial x^{i}}-\frac{d}{d t} \frac{\partial L}{\partial x_{(1)}^{i}}+\frac{d^{2}}{d t^{2}} \frac{\partial L}{\partial x_{(2)}^{i}}-\cdots+(-1)^{k} \frac{d^{k}}{d t^{k}} \frac{\partial L}{\partial x_{(k)}^{i}}\right) d t,
\end{aligned}
$$

где во всех выражениях, которые не находятся под интегралом, все производные $L$ взяты в точке $\left(\gamma^{i}(b), \ldots, \stackrel{(k)}{\gamma} i(b)\right)$ и производная $d / d t$ определена в (4.5). Напомним, что хорошо известная формула для уравнений Эйлера-Лагранжа для систем с высшими производными имеет следующий вид:

$$
\frac{\partial L}{\partial x^{i}}-\frac{d}{d t} \frac{\partial L}{\partial x_{(1)}^{i}}+\frac{d^{2}}{d t^{2}} \frac{\partial L}{\partial x_{(2)}^{i}}-\cdots+(-1)^{k} \frac{d^{k}}{d t^{k}} \frac{\partial L}{\partial x_{(k)}^{i}}=0,
$$

что совпадает с подынтегральным выражением в (А.5). Следовательно, интеграл обращается в нуль, и мы получаем окончательную формулу:

$$
\begin{aligned}
& \delta S=h^{i}(b)\left(\frac{\partial L}{\partial x_{(1)}^{i}}-\frac{d}{d t} \frac{\partial L}{\partial x_{(2)}^{i}}+\cdots+(-1)^{k-1} \frac{d^{k-1}}{d t^{k-1}} \frac{\partial L}{\partial x_{(k)}^{i}}\right)+ \\
&+\dot{h}^{i}(b)\left(\frac{\partial L}{\partial x_{(2)}^{i}}-\frac{d}{d t} \frac{\partial L}{\partial x_{(3)}^{i}}+\cdots+(-1)^{k-2} \frac{d^{k-2}}{d t^{k-2}} \frac{\partial L}{\partial x_{(k)}^{i}}\right)+\cdots \\
& \cdots+h_{(k-1)}^{i}(b) \frac{\partial L}{\partial x_{(k)}^{i}} .
\end{aligned}
$$

ПРИЛОЖЕНИЕ Б

Трехпараметрическое семейство точек в $T^{3} \mathcal{M}$, соответствующее одной и той же экстремальной кривой

Заменим параметр $t$ вдоль кривой на $f(t)$, где $f$ - произвольная гладкая функция:

$$
\begin{gathered}
x \mapsto x, \quad \dot{x} \mapsto \frac{1}{\dot{f}(t)} \dot{x}, \quad \ddot{x} \mapsto \frac{1}{\dot{f}(t)^{2}} \ddot{x}-\frac{\ddot{f}(t)}{\dot{f}(t)^{3}} \dot{x}, \\
\dddot{x} \mapsto \frac{1}{\dot{f}(t)^{3}} \dddot{x}-3 \frac{\ddot{f}(t)}{\dot{f}(t)^{4}} \ddot{x}+\left(3 \frac{\ddot{f}(t)^{2}}{\dot{f}(t)^{5}}-\frac{\ddot{f}(t)}{\dot{f}(t)^{4}}\right) \dot{x} .
\end{gathered}
$$

Введем обозначения

$$
\alpha:=\frac{1}{\dot{f}\left(t_{0}\right)}, \quad \beta:=-\frac{\ddot{f}\left(t_{0}\right)}{\dot{f}\left(t_{0}\right)^{3}}, \quad \delta:=3 \frac{\ddot{f}\left(t_{0}\right)^{2}}{\dot{f}\left(t_{0}\right)^{5}}-\frac{\dddot{f}\left(t_{0}\right)}{\dot{f}\left(t_{0}\right)^{4}},
$$

где $t_{0}$ - значение параметра $t$ в точке $x_{0}$. Таким образом, заменяя $t \mapsto f(t)$, получим

$$
\left(x^{i}, \dot{x}^{i}, \ddot{x}^{i}, \dddot{x}^{i}\right) \mapsto\left(x^{i}, \alpha \dot{x}^{i}, \alpha^{2} \ddot{x}^{i}+\beta \dot{x}^{i}, \alpha^{3} \dddot{x}^{i}+3 \alpha \beta \ddot{x}^{i}+\delta \dot{x}^{i}\right) .
$$


Благодарности. Авторы благодарны В. Долотину, А. Миронову, А. Морозову, А. Пополитову, В. Рубцову и И. Тютину за плодотворные обсуждения и очень полезные замечания. Мы также благодарны участникам семинара В. Долотина и всем, кто обсуждал с нами эту работу. Данная работа была осуществлена при частичной поддержке Программы поддержки ведущих научных школ (грант НШ-8004.2006.2), РФФИ (гранты № 07-02-00645 (П. Дунин-Барковский) и № 07-02-00878 (А. Слепцов)).

\section{Список литературы}

[1] В. И. Арнольд, Математические методы классической механики, Эдиториал УРСС, M., 2003.

[2] В. И. Арнольд, А. Б. Гивенталь, Симплектическая геометрия, РХД, Ижевск, 2000.

[3] A. Kushner, V. Lychagin, V. Rubtsov, Contact Geometry and Nonlinear Differential Equations, Encyclopedia Math. Appl., 101, Cambridge Univ. Press, Cambridge, 2007.

[4] R. Miron, D. Hrimiuc, H. Shimada, S. V. Sabau, The Geometry of Hamilton and Lagrange Spaces, Fund. Theories Phys., 118, Kluwer, Dordrecht, 2001.

[5] A. Morozov, Hamiltonian formalism in the presence of higher derivatives, arXiv: 0712.0946.

[6] Б. А. Дубровин, С.П. Новиков, А.Т. Фоменко, Современная геометрия. Методы и приложения. Т. 2. Геометрия и топология многообразий, Эдиториал УРСС, М., 2001.

[7] Д. М. Гитман, И. В. Тютин, Каноническое квантование полей со связями, Наука, М., 1986.

[8] D. Fairlie, J. Govaerts, A. Morozov, Nucl. Phys. B, 373:1 (1992), 214-232; arXiv: hep-th/9110022.

[9] L. Takhtajan, Comm. Math. Phys., 160:2 (1994), 295-315; arXiv: hep-th/9301111.

[10] T. Curtright, C. Zachos, Phys. Rev. D, 68 (2003), 085001; arXiv: hep-th/0212267.

[11] C. Rovelli, Quantum Gravity, Cambridge Monogr. Math. Phys., Cambridge Univ. Press, 2004.

[12] J. Muñoz Masqué, L. M. Pozo Coronado, J. Phys. A, 31:29 (1998), 6225-6242.

[13] А. Д. Миронов, А. Ю. Морозов, ТМФ, $156: 2$ (2008), 282-291; arXiv: hep-th/0703097

[14] A. Mironov, A. Morozov, On the problem of radiation friction beyond 4 and 6 dimensions, arXiv: 0710.5676.

[15] D. Galakhov, Писъма в ЖЖЭТФ, 87:8 (2008), 522-527; arXiv: 0710.5688.

[16] M. S. Plyushchay, Internat. J. Modern Phys. A, 4:15 (1989), 3851-3865.

[17] Л. Д. Ландау, Е. М. Лифшиц, Теоретическая физика. Т. II. Теория поля, Физматлит, М., 2001.

Поступила в редакцию 13.02.2008, после доработки 31.03.2008

4 Теоретическая и математическая физика, т. 158, № 1, 2009 г. 